\title{
New perspectives of purple starthistle (Centaurea calcitrapa) leaf extracts: phytochemical analysis, cytotoxicity and antimicrobial activity
}

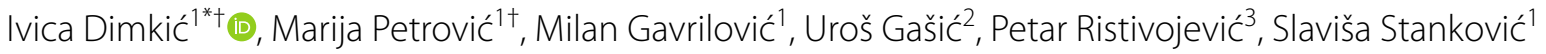
and Peđa Janaćkovićl

\begin{abstract}
Ethnobotanical and ethnopharmacological studies of many Centaurea species indicated their potential in folk medicine so far. However, investigations of different Centaurea calcitrapa L. extracts in terms of cytotoxicity and antimicrobial activity against phytopathogens are generally scarce. The phenolic profile and broad antimicrobial activity (especially towards bacterial phytopathogens) of methanol (MeOH), $70 \%$ ethanol (EtOH), ethyl-acetate (EtOAc), 50\% acetone $\left(\mathrm{Me}_{2} \mathrm{CO}\right)$ and dichloromethane: methanol $(\mathrm{DCM}: \mathrm{MeOH}, 1: 1)$ extracts of C. calcitrapa leaves and their potential toxicity on MRC-5 cell line were investigated for the first time. A total of 55 phenolic compounds were identified: 30 phenolic acids and their derivatives, 25 flavonoid glycosides and aglycones. This is also the first report of the presence of centaureidin, jaceidin, kaempferide, nepetin, flavonoid glycosides, phenolic acids and their esters in C. calcitrapa extracts. The best results were obtained with EtOAc extract with lowest MIC values expressed in $\mu \mathrm{g} / \mathrm{mL}$ ranging from 13 to 25, while methicillin resistant Staphylococcus aureus was the most susceptible strain. The most susceptible phytopathogens were Pseudomonas syringae pv. syringae, Xanthomonas campestris pv. campestris and Agrobacterium tumefaciens. The highest cytotoxicity was recorded for EtOAc and $\mathrm{Me}_{2} \mathrm{CO}$ extracts with the lowest relative and absolute $\mathrm{IC}_{50}$ values between 88 and $102 \mu \mathrm{g} / \mathrm{mL}$, while EtOH extract was the least toxic with predicted relative $\mathrm{IC}_{50}$ value of $1578 \mu \mathrm{g} / \mathrm{mL}$. Our results indicate that all tested extracts at concentration considered as non-toxic can be one of great importance in combat towards phytopathogenic and human pathogenic strains, as well as natural sources of antimicrobials.
\end{abstract}

Keywords: Centaurea calcitrapa, Phytochemistry, Liquid chromatography-mass spectrometry, Metabolite profile, New antimicrobials, Cytotoxicity

\section{Introduction}

In the last few decades, there have been an increased number of resistant pathogens globally due to uncontrolled use of antibiotics for medical and agricultural purposes. On the other hand, overuse of artificial chemical

\footnotetext{
*Correspondence: ivicad@bio.bg.ac.rs

†Ivica Dimkić and Marija Petrović contributed equally to this work

${ }^{1}$ Faculty of Biology, University of Belgrade, Studentski trg 16,

11000 Belgrade, Serbia

Full list of author information is available at the end of the article
}

pesticides has led to the formation of multidrug-resistant phytopathogenic strains. The spread of multidrug-resistant strains brings a new generation of diseases that cannot be treated with existing agents. Pesticides, which are widely used for suppression of plant pathogens as well as to insure and increase crop yields, are considered as environmental pollutants and provokers of animal and human health implications. Because of that, there are increasingly seeking for natural components as an alternative to these agents. Plants are well known as a
Springer Open

(c) The Author(s) 2020. This article is licensed under a Creative Commons Attribution 4.0 International License, which permits use, sharing, adaptation, distribution and reproduction in any medium or format, as long as you give appropriate credit to the original author(s) and the source, provide a link to the Creative Commons licence, and indicate if changes were made. The images or other third party material in this article are included in the article's Creative Commons licence, unless indicated otherwise in a credit line to the material. If material is not included in the article's Creative Commons licence and your intended use is not permitted by statutory regulation or exceeds the permitted use, you will need to obtain permission directly from the copyright holder. To view a copy of this licence, visit http://creativeco mmons.org/licenses/by/4.0/. 
powerful natural source of metabolites that can be used in combating resistant strains through different mechanisms (Margni et al. 2002; Moghannem et al. 2016; Shin et al. 2018).

The genus Centaurea is one of the largest genera in the family Asteraceae which contains approximately 400-700 species (Garcia-Jacas et al. 2001). It belongs to the subtribe Centaureinae (Cass.) Dumort of the tribe Cardueae Cass. Members of the genus Centaurea are distributed all around the world, especially in Mediterranean area and Western Asia (Sussana and Garcia-Jacas, 2007). In Serbia, 32 species are represented (Gajić 1975). Ethnobotanical and ethnopharmacological studies showed that many Centaurea species have been used in folk medicine for treatment of various diseases (Khammar and Djeddi 2012). Centaurea species had been used for medical purposes (antidiarrheal, anti-inflammatory, antirheumatic, antipyretic, antibacterial and cytotoxic) for hundreds of years. Traditionally, leaves and shoots of $C$. triumfettii All., C. urvillei DC. spp. stepposa Wagenitz, C. pullata L. and C. calcitrapa L. are used in diet, either as raw material or in processed form, and some of the species are used for making beverages and tonics (Lentini 2000; Pieroni et al. 2002; Khammar and Djeddi 2012). Species such as C. jacea L., C. calcitrapa, C. uniflora Turra, are used to reduce fever; C. behen L. and C. calcitrapa for treatment of jaundice; C. cyanus L. and C. ornata Willd., for improving circulation; C. ornata and C. sadleriana Janka, in veterinary medicine; C. cyanus and C. jacea for appetite improvement (Arif et al. 2004; Csupor et al. 2010; Khammar and Djeddi 2012). Phytochemical studies of Centaurea species have shown the presence of numerous natural compounds that exhibit different biological activities (Khammar and Djeddi 2012; Borges et al. 2013; Xie et al. 2015). The presence of natural components such as sesquiterpene lactones, phenolic acids, flavonoids and steroids favors the genus Centaurea over other genera within the Asteraceae family in terms of extended biological activities (Dumlu and Gürkan 2006).

C. calcitrapa is a biennial, herbaceous plant up to $60 \mathrm{~cm}$ high, commonly known as purple starthistle (Fig. 1). Flower heads (capitulum inflorescence) of this species are sessile, arranged laterally and at the top of the shoot with many bright purple-red tubular flowers. Involucre is ovoid, consisting of many phyllaries of which external are with long, yellow, terminal spine distinctive for this species. It is widespread in the western and southern parts of central Europe, North Africa and Western Asia to Northwestern India. It grows along roads, in waste places, between the rails and prefers rocky places, arable land, sunny and warm slopes (Mohlenbrock 2015). In the Southern Italy (Vulture area), young shoots of C. calcitrapa are consumed, as boiled and fried in mixtures with other weedy non-cultivated herbaceous plants (Pieroni et al. 2002). Traditionally, it was used for the treatment of ophthalmia, common fever, jaundice, digestive and skin disorders (Sarker et al. 2005; Csupor et al. 2010). Previous investigations on different extracts from this plant have shown certain bioactivity potential. A strong antioxidant activity has been reported for aqueous and methanol $(\mathrm{MeOH})$ extracts and significant cytotoxic activity for the $\mathrm{MeOH}$ extract on HeLa (human cervix adenocarcinoma) and Vero (epithelial cells of African green monkey kidney) cell lines (Erol-Dayi et al. 2011). The MeOH extract has shown a strong antibacterial activity towards certain pathogens including species of Bacillus, Pseudomonas, Staphylococcus, Streptococcus, Salmonella, Enterobacter, Enterococcus, Acinetobacter, and Escherichia genera (Toribio et al. 2004; Soumia et al. 2014; Moghannem et al. 2016). Several phytochemical studies revealed sterols, sesquiterpene lactones and their closely related group of triterpenoids, bisabolenes, lignans and flavonoids as constituents of C. calcitrapa extracts (Karawya et al. 1975; Dawidar et al. 1989; Al-Easa and Rizk 1992; Marco et al.

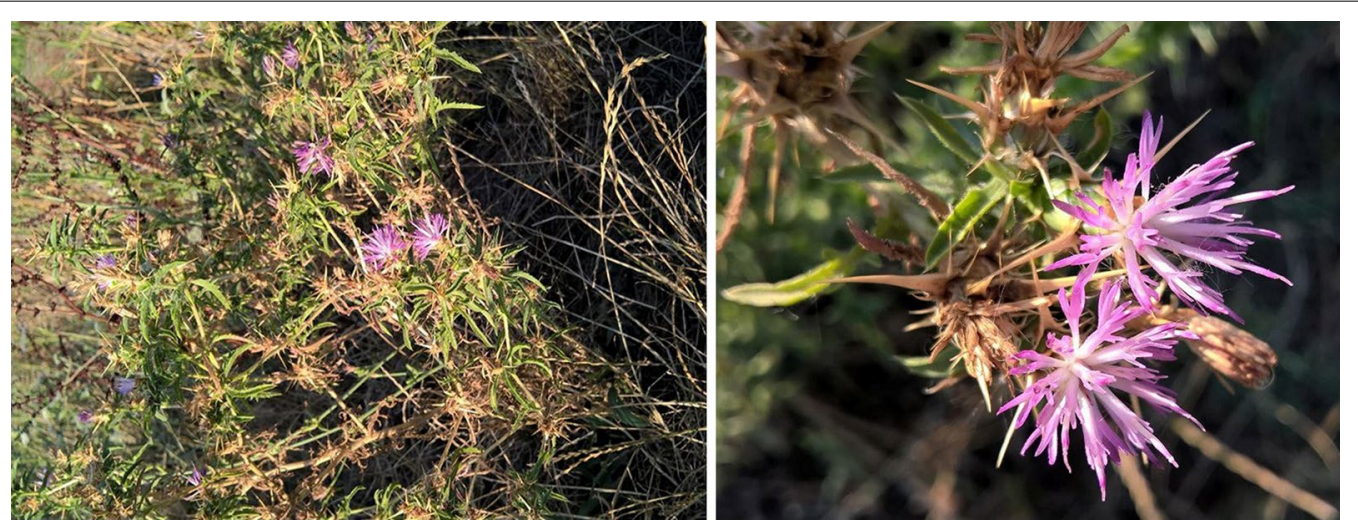

Fig. 1 Centaurea calcitrapa L. (village Sakule, Opovo municipality, the South Banat District, Vojvodina province, Serbia) 
1992; Formisano et al. 2012; Bruno et al. 2013; Kitouni et al. 2015).

To the best of our knowledge, there are no study reports of the cytotoxicity on MRC-5 cells and antimicrobial activity of $C$. calcitrapa extracts, particularly towards phytopathogenic bacteria. The main objective of the present study was to determine and evaluate phenolic profiles and antimicrobial potential of different $C$. calcitrapa leaf extracts against Candida albicans, as well as on human and phytopathogenic bacterial strains. In addition, cytotoxicity of different extracts was analyzed. Multidisciplinary approach was applied as an important tool for proposing new biological antimicrobial products that positively affects human health and reduces antibacterial resistance.

\section{Material and methods}

\section{Plant material}

Aerial parts of $C$. calcitrapa were collected during the flowering season from a village Sakule, (Opovo municipality, the South Banat District, Vojvodina province, Serbia, N $45^{\circ} 08^{\prime} 14.5^{\prime \prime}$, E $20^{\circ} 27^{\prime} 55.8^{\prime \prime}$ ) in July 2015 . The plant material was authenticated, following professional literature for determination (Gajić 1975; Javorka and Csapody 1975). The voucher specimen was deposited in the Herbarium of the University of Belgrade-Faculty of Biology, Institute of Botany and Botanical Garden "Jevremovac" (BEOU 17509).

\section{Indicator strains and growth conditions}

Antimicrobial activity of different extracts was tested against one yeast (Candida albicans ATCC 10231), five human opportunistic and pathogenic bacterial strains (Escherichia coli ATCC 25922, Enterococcus faecalis ATCC 29212, Listeria monocytogenes ATCC 19111, Staphylococcus aureus ATCC 25923 and methicillin resistant Staphylococcus aureus (MRSA) ATCC 33591); and nine phytopathogens (Agrobacterium tumefaciens, Erwinia amylovora NCPPB 683, Pseudomonas syringae pv. aptata (P16, P29, and P49), Pseudomonas syringae pv. syringae GSPB 1142, Xanthomonas campestris pv. campestris NCPPB 583, Xanthomonas arboricola pv. juglandis (IZB 320 and IZB 321). For differential strains growth, Luria-Bertani medium (LB) (Thermo Fisher Scientific, USA) was used for all strains, with the exception for $L$. monocytogenes and C. albicans which were cultured in Brain Heart Infusion broth (BHI) (HiMedia, India) and in Trypticase soy broth (TSB) (LAB M Ltd., UK), respectively. The human and phytopathogenic strains were cultured overnight at $37^{\circ} \mathrm{C}$ and $30{ }^{\circ} \mathrm{C}$, respectively. All reference strains used belonged to the Department of Microbiology, Faculty of Biology, University of Belgrade, while phytopathogenic strains, previously identified, belong to the collection of the Institute for Plant Protection and Environment, Belgrade, Serbia. All strain suspensions for antimicrobial testing were adjusted to McFarland standard turbidity (0.5), which corresponds to $10^{7}-10^{8} \mathrm{CFU} / \mathrm{mL}$.

\section{Cell culture}

Fetal lung fibroblasts MRC- 5 cells (ATCC CCL-171) were grown in RPMI 1640 medium supplemented with $10 \%$ FBS, $100 \mathrm{U} / \mathrm{mL}$ penicillin/streptomycin and $2 \mathrm{mM} \mathrm{L-glu-}$ tamine. Cells were maintained in a humidified atmosphere containing $5 \% \mathrm{CO}_{2}$ at $37{ }^{\circ} \mathrm{C}$ and subcultured at $90 \%$ confluence, twice each week, using $0.05 \%$ trypsin-EDTA. Cell viability was determined by the trypan blue dye exclusion method.

\section{Extraction of phenolic compounds}

Extraction of specialized metabolites was performed by maceration combined with ultrasonic extraction. Plant material, $10 \mathrm{~g}$ of air-dried C. calcitrapa leaves, was milled into powder for 5 min using a laboratory mill and submerged in $150 \mathrm{~mL}$ of a given solvent or solvent mixture: methanol $(\mathrm{MeOH}), 70 \%$ ethanol $(\mathrm{EtOH})$, ethyl-acetate (EtOAc), 50\% acetone $\left(\mathrm{Me}_{2} \mathrm{CO}\right)$ and dichloromethane: methanol (DCM: $\mathrm{MeOH}=1: 1$ ). Five different samples were then ultrasonicated for $30 \mathrm{~min}$ in the ultrasonic bath at $25^{\circ} \mathrm{C}$, and filtered through a syringe filter (PTFE 120 membrane, $0.45 \mu \mathrm{m}$, Supelco, Bellefonte, PA, USA), evaporated to dryness $\left(45^{\circ} \mathrm{C}\right)$ using rotary vacuum evaporator and extracts were dissolved in $\mathrm{MeOH}$ at final concentration of $30 \mathrm{mg} / \mathrm{mL}$. The obtained methanolic solutions were stored at $-20{ }^{\circ} \mathrm{C}$ prior to analysis. The extraction yield of obtained extracts was determined according to the equation described in Janaćković et al. (2019) and expressed in percentages as a quotient of dry weight of the extracts and mass of dried plant material.

\section{UHPLC-LTQ Orbitrap-MS ${ }^{4}$}

For the analysis, a $1000 \mathrm{mg} / \mathrm{L}$ stock solution of a mixture of phenolic compounds (Sigma-Aldrich Steinheim, Germany) was prepared by dissolving in $\mathrm{MeOH}$ (HPLC grade, Merck Darmstadt, Germany). Working solutions (0.025; 0.050; 0.100; 0.250; 0.500; 0.750 and $1.000 \mathrm{mg} / \mathrm{L}$ ) were prepared by diluting the stock solution with mobile phase (acetonitrile $(\mathrm{ACN}): \mathrm{H}_{2} \mathrm{O}=1: 1$ ). Calibration curves were obtained by plotting the peak areas of the standards against their concentration. Ultra-pure water (Thermofisher TKA, MicroPure water purification system, $0.055115 \mu \mathrm{S} / \mathrm{cm}$ ) was used to prepare standard solutions and blanks. The analytical separation was performed on a Syncronis C18 column $(50 \times 2.1 \mathrm{~mm}$, $1.7 \mu \mathrm{m}$ particle size; Thermo Fisher Scientific, USA). The mobile phase consisted of (A) $0.1 \%$ MS grade formic acid 
(Merck Darmstadt, Germany) aqueous solution and (B) $0.1 \%$ formic acid in MS grade ACN (Merck Darmstadt, Germany). The gradient program was as follows: 0.0$1.0 \mathrm{~min}, 5 \% \mathrm{~B} ; 1.0-14.0 \mathrm{~min}, 5-95 \% \mathrm{~B} ; 14.0-14.1 \mathrm{~min}$, 95-5\% B; 14.1-20.1 $\mathrm{min}, 5 \% \mathrm{~B}$. The injection volume for all samples was $5 \mu \mathrm{L}$ and the flow rate was $275 \mu \mathrm{L}$ per $\mathrm{min}$. The UHPLC system was coupled to a linear ion trap and Orbitrap hybrid mass spectrometer (LTQ Orbitrap XL) equipped with a heated-electrospray ionization probe (HESI-II, Thermo Fisher Scientific, Germany). The system was operated in negative ionization mode with the following conditions: source voltage at $5 \mathrm{kV}$, capillary voltage at $-40 \mathrm{~V}$, tube lens voltage at $-80 \mathrm{~V}$, capillary temperature at $275{ }^{\circ} \mathrm{C}$, sheath and auxiliary gas $\left(\mathrm{N}_{2}\right)$ with a flow rate of 42 and 11 arbitrary units. The mass spectra were recorded from 100 to $900 \mathrm{~m} / \mathrm{z}$. Collision-induced dissociation (CID) was used for the study of fragmentation of the tested compounds. The normalized collision energy of the CID cell was constant and was $35 \mathrm{eV}$ (Banjanac et al. 2017).

Phenolics were identified and quantified by comparison with the retention time, accurate mass and the mass spectra of standard compounds. Tentative identification of unknown compounds (in the absence of standards) were done on the basis of their monoisotopic mass (obtained by full scan analysis) and $\mathrm{MS}^{4}$ fragmentation and confirmed using previously reported NMR and MS fragmentation data about Centaurea species found in literature (Flamini et al. 2001; Forgo et al. 2012; Bakr et al. 2016; Zengin et al. 2018). ChemDraw software (version 12.0, CambridgeSoft, Cambridge, MA, USA) was used to calculate accurate mass of compounds of interest. In this way, molecular formulas of unknown compounds were obtained, while their identification was suggested according to specific MS fragmentation data.

\section{Principal component analysis}

Principal component analysis (PCA) was performed using the PLS Toolbox software package (Eigenvector Re- search, Inc., Manson, WA, USA) for MATLAB (Version 7.12.0 R2011a), PCA was carried out as an exploratory data analysis by using singular value decomposition (SVD) algorithm.

\section{Antimicrobial assays Well diffusion test}

The initial screening test of antimicrobial activity of different extracts was determined by a modified well-diffusion method as described by Dimkić et al. (2016). Sterile molds for the wells were placed on the Plate Count (PC) Agar (bioMérieux, France) which was used as the solid medium and $6 \mathrm{~mL}$ of LA/TSA/BHI soft agar, previously inoculated with $60 \mu \mathrm{L}$ (McFarland 0.5 turbidity) of the appropriate strain, was uniformly spread on the PC agar. After the soft agar solidification, into the resulting wells $20 \mu \mathrm{L}$ of each extract $(30 \mathrm{mg} / \mathrm{mL})$ was added to the wells. As a negative control, $\mathrm{MeOH}$ was used, while fungicide nystatin and antibiotic vancomycin (both in $200 \mu \mathrm{g} / \mathrm{mL}$ concentrations) were used as positive controls. The Petri plates were incubated for $24 \mathrm{~h}$ at the optimal temperature $\left(30^{\circ} \mathrm{C}\right.$ and $\left.37^{\circ} \mathrm{C}\right)$ for indicator strains. Diameter of growth inhibition zones was expressed in $\mathrm{mm}$.

\section{MIC assay}

To determine the minimum inhibitory concentration (MIC) and minimum bactericidal concentration (MBC) for different $C$. calcitrapa leaf extracts, the broth microdilution method as described by Dimkić et al. (2016) was used. Two-fold serial dilutions with LB medium in 96-well microtiter plates were performed. The concentrations of tested extracts were in the range from 8-1000 $\mu \mathrm{g} /$ $\mathrm{mL}$. Besides a negative control (bacterial growth control), a sterility control, and a control for the solvent $(\mathrm{MeOH})$, the antibiotics streptomycin and vancomycin (Sigma Aldrich, Steinheim, Germany) were also tested as positive controls. Concentration of the antibiotics was in the range from $1-400 \mu \mathrm{g} / \mathrm{mL}$, while the solvent (absolute methanol) was $10 \%$. All dilutions were done in triplicate. Each well, except for the sterility control, was inoculated with $20 \mu \mathrm{L}$ of bacterial culture (approx. $1 \times 10^{7} \mathrm{CFU} / \mathrm{mL}$ ), reaching a final volume of $200 \mu \mathrm{L}$. Lastly, $22 \mu \mathrm{L}$ of resazurin [Resazurin Sodium Salt TCI, Belgium (>90\% (LC) $\left.\left.\mathrm{C}_{12} \mathrm{H}_{6} \mathrm{NNaO}_{4}=251.17 \mathrm{~g} / \mathrm{mol}\right)\right]$, at a final concentration of $675 \mu \mathrm{g} / \mathrm{mL}$, was added to each well. The plates were incubated overnight at $30{ }^{\circ} \mathrm{C}$ and $37{ }^{\circ} \mathrm{C}$ for phytopathogenic and human pathogens, respectively. All tests were performed in a lighted environment, but the plates were incubated in the dark. The assay is based on the ability of metabolically active cells to reduce non-fluorescent resazurin to pink and fluorescent resorufin and finally to colorless dihydroresorufin by enzymes oxidoreductases within viable cells (Karuppusamy and Rajasekaran 2009). The lowest concentration which showed no change in color was defined as the MIC. MBC was determined by sub-culturing the test dilutions from each well without color change on agar plates and incubating for $24 \mathrm{~h}$. The lowest concentration without bacterial growth was defined as the MBC value. The results are expressed in $\mu \mathrm{g} / \mathrm{mL}$.

\section{Cytotoxicity assay}

The cytotoxic effect of the plant extracts was assessed by modified MTT assay (Mosmann 1983). Briefly, MRC-5 cells were seeded into 96-well plates at a density of $2 \times 10^{4}$ cells/well and incubated overnight at $5 \%$ $\mathrm{CO}_{2}, 37^{\circ} \mathrm{C}$. After forming a monolayer, the medium was 
removed and replaced with fresh medium containing test substances (concentration range was 3.9-250 $\mu \mathrm{g} / \mathrm{mL}$ ) and incubation continued for $24 \mathrm{~h}$. Next day, MTT solution (final concentration $500 \mu \mathrm{g} / \mathrm{mL}$ ) was added and cells were incubated for an additional $3 \mathrm{~h}$. The formed formazan crystals were dissolved in DMSO. Cell viability was determined by measuring absorbance (A) at $570 \mathrm{~nm}$ using a microplate reading spectrophotometer (Multiskan FC, Thermo Scientific, Shanghai, China). Survival percentage (S) was calculated using the following formula:

$$
S(\%)=100 *\left(\frac{A_{\text {test substance }}}{A_{\text {control }}}\right)
$$

The concentration of the tested compound that exerts half of its maximal inhibitory effect of cell viability $\left(\mathrm{IC}_{50}\right)$ was also determined. Quest $\mathrm{Graph}^{\mathrm{TM}} \mathrm{IC}_{50}$ Calculator (AAT Bioquest Inc. 2009) calculator was used to generate absolute and relative $\mathrm{IC}_{50}$ values for each extract. The analysis of variance was supported by the KolmogorovSmirnov test for the normality of residuals and obtained data were subjected to the variance analysis (One-way ANOVA). The means separation of cell survival values were accomplished by Tukey's HSD (honest significant difference) test. The significance level was evaluated at $P<0.05$. Statistical analyses were conducted using general procedures of STATISTICA v.7 (StatSoft, Inc.) and IBM SPSS Statistics v.20 (SPSS, Inc.).

\section{Results}

\section{Extraction yield}

In this study, the influence of different solvents $(\mathrm{MeOH}$, $70 \% \mathrm{EtOH}$, EtOAc, 50\% $\mathrm{Me}_{2} \mathrm{CO}$ and DCM: $\mathrm{MeOH}=1: 1$ ) on the yield of extraction were examined. The yields of extraction by obtained extracts increased in the following order: $\mathrm{EtOAc}<\mathrm{DCM}: \mathrm{MeOH}<\mathrm{MeOH}<70 \% \mathrm{EtOH}<50 \%$ $\mathrm{Me}_{2} \mathrm{CO}$. The lowest yield was obtained by EtOAc (9.4\%). Similar yield was observed for $70 \% \mathrm{EtOH}$ and $50 \%$ $\mathrm{Me}_{2} \mathrm{CO}$ extract (23.3\% and 23.8\%, respectively). Moderate yield was scored for $\mathrm{MeOH}$ and DCM: $\mathrm{MeOH}(16.6 \%$ and $13.3 \%$, respectively).

\section{UHPLC-LTQ Orbitrap MS ${ }^{4}$}

Incorporating $\mathrm{LC}-\mathrm{MS}^{4}$ in an offline fashion as demonstrated in current study, allowed the identification of 55 plant metabolites: as 30 phenolic acids and their derivatives, as well as 25 flavonoid glycosides and aglycones (Table 1). Sixteen compounds were confirmed using the available commercial standards (Additional file 1: Table S1), while the others were tentatively identified by the examination of exact masses of unknown compounds and its $\mathrm{MS}^{4}$ fragmentations. The yields of quantified phenolics in the tested extracts can be represented as follows: EtOAc $<\mathrm{MeOH}<\mathrm{DCM}: \quad \mathrm{MeOH}<\mathrm{EtOH}<\mathrm{Me}_{2} \mathrm{CO}$. The most abundant phenolic acids in investigated extracts were hydrocinnamic acid derivatives, which were found in free form and as glycosides, and also as quinic acid esters. The phenolic acids and their derivatives (1-30) share a common fragmentation pathway based on loss of the $\mathrm{CO}_{2}$ group resulting in $\left[\mathrm{M}-\mathrm{H}-\mathrm{CO}_{2}\right]^{-},-44 \mathrm{Da}$ (Ristivojević et al. 2015). Phenolic acid derivatives (1113 and 15), showed the main fragmentation mechanism based on homolytic cleavage of the ester moiety yielding a negative product ion of corresponding phenolic acid. For example, compound $\mathbf{1 8}$ showed a molecular ion at $\mathrm{m} / z 355$ and was assigned to be the deprotonated molecule $[\mathrm{M}-\mathrm{H}]^{-}$of feruloyl hexoside. The $\mathrm{MS}^{2}$ base peak of compound 18 at $193 \mathrm{~m} / z$ (deprotonated ferulic acid) was formed by loss of hexosyl group-162 Da. $\mathrm{MS}^{3}$ and $\mathrm{MS}^{4}$ base peaks at 149 and $134 \mathrm{~m} / z$ corresponding to further loss of $\mathrm{CO}_{2}(18 \mathrm{Da})$ and methyl group $(15 \mathrm{Da})$, respectively.

All identified flavonoid aglycones were from the flavone subgroup (apigenin, luteolin, scutellarein, chrysoeriol, nepetin, jaceosidin, and eupatorin) and flavonol subgroup (kaempferol, kaempferide, jaceidin and centaureidin), while the glycosides were found to be flavone 7- $O$ derivatives and flavonol 3- $O$ derivatives. Compounds 51 and 55 with same quasimolecular ions at $359 \mathrm{~m} / z$ and retention times at 9.74 and $11.24 \mathrm{~min}$, respectively, were identified by examination of its fragmentation pattern. Both compounds produced the same $\mathrm{MS}^{2}$ base peak at $344 \mathrm{~m} / z$ (loss of methyl group-15 Da), but different $\mathrm{MS}^{3}$ and $\mathrm{MS}^{4}$ fragmentation. In the case of compounds 55, MS ${ }^{3}$ base peak at $301 \mathrm{~m} / z$ was formed by the loss of $43 \mathrm{Da}\left(\mathrm{CH}_{3}+\mathrm{CO}\right)$, and this compound was marked as centaureidin, while the compounds $\mathbf{5 1}$ was marked as jaceidin, positional isomer of centaureidin. Proposed fragmentation pathways of these isomeric compounds are depicted in Fig. 2. Furthermore, eight phenolic acids (derivatives of hydroxybenzoic acids: $p$-hydroxybenzoic, protocatechuic, gallic, gentisic acid; derivatives of hydroxycinnamic acids: $p$-coumaric, ferulic, caffeic, chlorogenic acid), four flavonoid aglycones (kaempferol, apigenin, luteolin, chrysoeriol), three flavonoid glycosides (quercetin 3-O-glucoside, isorhamnetin 3-O-glucoside, kaempferol 3-O-glucoside) and coumarin derivative (aesculetin) were quantified (Additional file 1: Table S1). Chlorogenic acid was the most abundant of all detected compounds, with the highest amount in EtOH extract. Among the quantified hydroxybenzoic acid derivatives, $p$-hydroxybenzoic acid was the most abundant, with the highest amount in DCM: $\mathrm{MeOH}$ extract. The highest concentrations of protocatechuic acid, caffeic acid and $p$-coumaric acid were obtained with $\mathrm{Me}_{2} \mathrm{CO}$ extract, while the highest amount of ferulic acid was recorded in $\mathrm{MeOH}$ extract. However, with the same concentration 
Table 1 Proposed metabolites in five different C. calcitrapa extracts using UPLC-MS/MS ${ }^{4}$ analysis

\begin{tabular}{|c|c|c|c|c|c|c|c|c|c|}
\hline No & $t_{\mathrm{R}^{\prime}} \min$ & $\begin{array}{l}\text { Compound } \\
\text { name }\end{array}$ & $\begin{array}{l}\text { Molecular } \\
\text { formula, } \\
{[\mathrm{M}-\mathrm{H}]^{-}}\end{array}$ & $\begin{array}{l}\text { Calculated } \\
\text { mass, } \\
{[\mathrm{M}-\mathrm{H}]^{-}}\end{array}$ & $\begin{array}{l}\text { Exact mass, } \\
{[\mathrm{M}-\mathrm{H}]^{-}}\end{array}$ & $\Delta \mathrm{mDa}$ & $\begin{array}{l}\text { MS }^{2} \text { Fragments, } \\
\text { (\% Base Peak) }\end{array}$ & $\begin{array}{l}\text { MS }{ }^{3} \text { Fragments, } \\
\text { (\% Base Peak) }\end{array}$ & $\begin{array}{l}\text { MS }{ }^{4} \text { Fragments, } \\
\text { (\% Base Peak) }\end{array}$ \\
\hline \multicolumn{10}{|c|}{ Phenolic acids and derivatives } \\
\hline 1 & 2.35 & Gallic acid ${ }^{a}$ & $\mathrm{C}_{7} \mathrm{H}_{5} \mathrm{O}_{5}^{-}$ & 169.01425 & 169.01476 & -0.51 & $\mathbf{1 2 5}(100)$ & $\begin{array}{l}96(14), 79(4) \\
\mathbf{8 1}(100), 9741) \\
107(5)\end{array}$ & ND \\
\hline 2 & 3.98 & $\begin{array}{l}\text { Dihydroxyben- } \\
\text { zoyl hexoside }\end{array}$ & $\mathrm{C}_{13} \mathrm{H}_{15} \mathrm{O}_{9}^{-}$ & 315.07216 & 315.07303 & -0.87 & $\begin{array}{l}\text { 109(13), 152(45), } \\
\mathbf{1 5 3 ( 1 0 0 )} \\
\text { 163(9), 165(12) }\end{array}$ & $108(4), \mathbf{1 0 9}(100)$ & $65(14), 81(100)$ \\
\hline 3 & 4.32 & $\begin{array}{l}\text { Protocatechuic } \\
\text { acid }^{a}\end{array}$ & $\mathrm{C}_{7} \mathrm{H}_{5} \mathrm{O}_{4}^{-}$ & 153.01933 & 153.02006 & -0.72 & 109(100), 110(5) & $\begin{array}{l}65(100), 75(8) \\
81(43), 227(11)\end{array}$ & ND \\
\hline 4 & 4.52 & $\begin{array}{l}\text { Dihydroxy- } \\
\text { benzoic acid } \\
\text { pentosyl- } \\
\text { hexoside }\end{array}$ & $\mathrm{C}_{18} \mathrm{H}_{23} \mathrm{O}_{13}^{-}$ & 447.11442 & 447.11586 & -1.45 & $\begin{array}{c}\text { 152(100), } \\
163(65) \\
177(35) \\
179(32) \\
207(35)\end{array}$ & 108(100), 124(3) & ND \\
\hline 5 & 4.60 & $\begin{array}{l}\text { Caffeoyl-quinic } \\
\text { acid isomer }\end{array}$ & $\mathrm{C}_{16} \mathrm{H}_{17} \mathrm{O}^{-}$ & 353.08781 & 353.08892 & -1.12 & $\begin{array}{l}\text { 135(6), 179(31), } \\
\text { 180(3), } \\
\mathbf{1 9 1 ( 1 0 0 )} \\
192(5)\end{array}$ & $\begin{array}{l}\text { 109(28), 111(41), } \\
\mathbf{1 2 7}(100) \\
\text { 171(26), } \\
173(69)\end{array}$ & $\begin{array}{l}81(7), 83(17), \\
\mathbf{8 5}(100), 99(33), \\
109(28)\end{array}$ \\
\hline 6 & 4.64 & $\begin{array}{l}\text { Feruloyl-quinic } \\
\text { acid hexoside } \\
\text { isomer } 1\end{array}$ & $\mathrm{C}_{23} \mathrm{H}_{29} \mathrm{O}_{14}^{-}$ & 529.15628 & 529.16127 & -4.99 & $\begin{array}{l}\text { 173(62), } \\
\text { 191(100), } \\
\text { 193(10), } \\
337(17) \\
367(74)\end{array}$ & $\begin{array}{l}\text { 85(81), 93(51), } \\
99(21), 109(20), \\
\mathbf{1 2 7}(100)\end{array}$ & $\begin{array}{l}81(28), \mathbf{8 3}(100) \\
85(41), 99(20) \\
109(27)\end{array}$ \\
\hline 7 & 4.71 & $\begin{array}{l}\text { Caffeoyl-hexaric } \\
\text { acid }\end{array}$ & $\mathrm{C}_{15} \mathrm{H}_{15} \mathrm{O}_{11}^{-}$ & 371.06199 & 371.06311 & -1.13 & $\begin{array}{l}\text { 163(15), 191(17), } \\
\text { 209(100), } \\
323(10) \\
325(17)\end{array}$ & $85(24), \mathbf{1 9 1}(100)$ & $\begin{array}{l}85(100), 111(6) \\
\quad 147(12), 173(8)\end{array}$ \\
\hline 8 & 4.83 & $\begin{array}{l}\text { Caffeoyl-quinic } \\
\text { acid hexoside }\end{array}$ & $\mathrm{C}_{22} \mathrm{H}_{27} \mathrm{O}_{14}{ }^{-}$ & 515.14063 & 515.14317 & -2.54 & 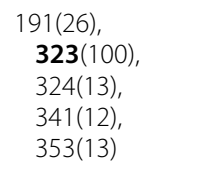 & 133(4), 161(100) & $117(18), 133(100)$ \\
\hline 9 & 5.15 & $\begin{array}{l}\text { Feruloyl-hexaric } \\
\text { acid }\end{array}$ & $\mathrm{C}_{16} \mathrm{H}_{17} \mathrm{O}_{11}^{-}$ & 385.07764 & 385.07870 & -1.07 & $\begin{array}{l}191(100), 209(5) \\
367(5)\end{array}$ & $\begin{array}{l}85(100), 129(7) \\
147(14), 173(4)\end{array}$ & $\mathbf{5 7}(100)$ \\
\hline 10 & 5.17 & $\begin{array}{l}\text { Coumaroyl- } \\
\text { quinic acid } \\
\text { isomer } 1\end{array}$ & $\mathrm{C}_{16} \mathrm{H}_{17} \mathrm{O}_{8}^{-}$ & 337.09289 & 337.09357 & -0.67 & 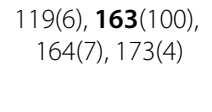 & $\mathbf{1 1 9}(100)$ & ND \\
\hline 11 & 5.24 & $\begin{array}{l}\text { Chlorogenic } \\
\text { acid }^{a}\end{array}$ & $\mathrm{C}_{16} \mathrm{H}_{17} \mathrm{O}_{9}^{-}$ & 353.08781 & 353.08813 & -0.32 & 179(3), 191(100) & $\begin{array}{l}\text { 85(100), 93(57), } \\
111(35) \\
127(89) \\
173(63)\end{array}$ & ND \\
\hline 12 & 5.26 & $\begin{array}{l}\text { Feruloyl-quinic } \\
\text { acid hexoside } \\
\text { isomer } 2\end{array}$ & $\mathrm{C}_{23} \mathrm{H}_{29} \mathrm{O}_{14}^{-}$ & 529.15628 & 529.15889 & -2.62 & $\begin{array}{l}\text { 175(29), } \\
\text { 191(100), } \\
\text { 193(8), 367(70) }\end{array}$ & $\begin{array}{l}\text { 85(95), 93(70), } \\
111(28) \\
\mathbf{1 2 7}(100) \\
173(53)\end{array}$ & $\begin{array}{l}81(25), 83(26), \\
\mathbf{8 5}(100), 99(37), \\
109(39)\end{array}$ \\
\hline 13 & 5.27 & $\begin{array}{l}p \text {-Coumaric } \\
\operatorname{acid}^{a}\end{array}$ & $\mathrm{C}_{9} \mathrm{H}_{7} \mathrm{O}_{3}^{-}$ & 163.04007 & 163.04072 & -0.65 & 119(100) & 119(100), 91(29) & ND \\
\hline 14 & 5.29 & $\begin{array}{l}\text { Coumaroyl } \\
\text { hexoside }\end{array}$ & $\mathrm{C}_{15} \mathrm{H}_{17} \mathrm{O}_{8}^{-}$ & 325.09289 & 325.09305 & -0.16 & $\begin{array}{c}119(9), \mathbf{1 6 3}(100) \\
164(8), 279(5)\end{array}$ & $\mathbf{1 1 9}(100)$ & ND \\
\hline 15 & 5.38 & $\begin{array}{l}\text { p-Hydroxyben- } \\
\text { zoic acid }\end{array}$ & $\mathrm{C}_{7} \mathrm{H}_{5} \mathrm{O}_{3}^{-}$ & 137.02442 & 137.02493 & -0.51 & $\begin{array}{l}\text { 93(100), 94(7), } \\
109(3)\end{array}$ & ND & ND \\
\hline 16 & 5.47 & $\begin{array}{r}\text { Feruloyl-quinic } \\
\text { acid isomer } 1\end{array}$ & $\mathrm{C}_{17} \mathrm{H}_{19} \mathrm{O}_{9}^{-}$ & 367.10346 & 367.10347 & -0.01 & $\begin{array}{l}\text { 134(6), } 193(100) \\
194(4)\end{array}$ & $\begin{array}{r}\text { 134(100), } \\
149(21)\end{array}$ & 106(100) \\
\hline 17 & 5.53 & Gentisic acid ${ }^{a}$ & $\mathrm{C}_{7} \mathrm{H}_{5} \mathrm{O}_{4}^{-}$ & 153.01933 & 153.01991 & -0.57 & 109(100), 110(7) & $\begin{array}{l}81(100), 90(20) \\
95(12), 168(13) \\
198(15)\end{array}$ & ND \\
\hline
\end{tabular}


Table 1 (continued)

\begin{tabular}{|c|c|c|c|c|c|c|c|c|c|}
\hline No & $t_{R^{\prime}} \min$ & $\begin{array}{l}\text { Compound } \\
\text { name }\end{array}$ & $\begin{array}{l}\text { Molecular } \\
\text { formula, } \\
{[\mathrm{M}-\mathrm{H}]^{-}}\end{array}$ & $\begin{array}{l}\text { Calculated } \\
\text { mass, } \\
{[\mathrm{M}-\mathrm{H}]^{-}}\end{array}$ & $\begin{array}{l}\text { Exact mass, } \\
{[\mathrm{M}-\mathrm{H}]^{-}}\end{array}$ & $\Delta \mathrm{mDa}$ & $\begin{array}{l}\text { MS }^{2} \text { Fragments, } \\
\text { (\% Base Peak) }\end{array}$ & $\begin{array}{l}\text { MS }^{3} \text { Fragments, } \\
\text { (\% Base Peak) }\end{array}$ & $\begin{array}{l}\text { MS }{ }^{4} \text { Fragments, } \\
\text { (\% Base Peak) }\end{array}$ \\
\hline 18 & 5.54 & $\begin{array}{l}\text { Feruloyl hexo- } \\
\text { side }\end{array}$ & $\mathrm{C}_{16} \mathrm{H}_{19} \mathrm{O}_{9}^{-}$ & 355.10346 & 355.10367 & -0.21 & $\begin{array}{l}191(8), \mathbf{1 9 3}(100) \\
194(3)\end{array}$ & $\begin{array}{l}\text { 134(73), } \\
\text { 149(100), } \\
\text { 178(69) }\end{array}$ & 134(100) \\
\hline 19 & 5.76 & Aesculetin $^{a}$ & $\mathrm{C}_{9} \mathrm{H}_{5} \mathrm{O}_{4}^{-}$ & 177.01933 & 177.01989 & -0.56 & $\begin{array}{l}\text { 131(5), 133(18), } \\
\text { 134(9), } \\
\text { 135(100), } \\
147(4)\end{array}$ & $\begin{array}{l}91(100), 93(12) \\
\text { 107(96), } \\
\text { 180(10), } \\
195(10)\end{array}$ & ND \\
\hline 20 & 5.76 & Caffeic acid ${ }^{a}$ & $\mathrm{C}_{9} \mathrm{H}_{7} \mathrm{O}_{4}^{-}$ & 179.03498 & 179.03552 & -0.54 & $\begin{array}{l}\text { 89(3), 133(2), } \\
\text { 135(100), } \\
\text { 136(8) }\end{array}$ & $\begin{array}{l}\text { 93(15), 106(6), } \\
\text { 107(100) } \\
117(24) \\
135(35)\end{array}$ & ND \\
\hline 21 & 5.89 & $\begin{array}{l}\text { Coumaroyl- } \\
\text { quinic acid } \\
\text { isomer } 2\end{array}$ & $\mathrm{C}_{16} \mathrm{H}_{17} \mathrm{O}_{8}^{-}$ & 337.09289 & 337.09279 & 0.10 & $\begin{array}{l}\text { 163(5), 173(11), } \\
\text { 191(100) } \\
192(3)\end{array}$ & $\begin{array}{l}85(100), 93(50) \\
111(38) \\
127(90) \\
173(62)\end{array}$ & $\begin{array}{l}\mathbf{5 3}(100), 75(76) \\
\quad 100(85), 113(85), \\
176(85)\end{array}$ \\
\hline 22 & 6.18 & $\begin{array}{r}\text { Feruloyl-quinic } \\
\text { acid isomer } 2\end{array}$ & $\mathrm{C}_{17} \mathrm{H}_{19} \mathrm{O}_{9}^{-}$ & 367.10346 & 367.10277 & 0.69 & $\begin{array}{l}\text { 173(25), } \\
\mathbf{1 9 1}(100) \\
192(4), 193(5)\end{array}$ & $\begin{array}{l}\text { 85(100), 93(61), } \\
111(36) \\
127(82) \\
173(65)\end{array}$ & $\begin{array}{l}\mathbf{5 7}(100), 70(19) \\
81(19), 167(22)\end{array}$ \\
\hline 23 & 6.30 & $\begin{array}{l}\text { Coumaroyl- } \\
\text { quinic acid } \\
\text { isomer } 3\end{array}$ & $\mathrm{C}_{16} \mathrm{H}_{17} \mathrm{O}_{8}^{-}$ & 337.09289 & 337.09246 & 0.43 & 163(4), 191(100) & $\begin{array}{l}\text { 85(100), 93(55), } \\
111(43) \\
127(95) \\
173(71)\end{array}$ & ND \\
\hline 24 & 6.49 & $\begin{array}{r}\text { Feruloyl-quinic } \\
\text { acid isomer } 3\end{array}$ & $\mathrm{C}_{17} \mathrm{H}_{19} \mathrm{O}_{9}^{-}$ & 367.10346 & 367.10371 & -0.26 & $\begin{array}{l}\text { 191(100), 192(3), } \\
193(3)\end{array}$ & $\begin{array}{l}\text { 85(100), 93(70), } \\
111(33) \\
127(96) \\
173(68)\end{array}$ & $\begin{array}{l}\text { 57(100), 80(28), } \\
148(31), 157(27), \\
162(37)\end{array}$ \\
\hline 25 & 6.82 & Ferulic acid ${ }^{a}$ & $\mathrm{C}_{10} \mathrm{H}_{9} \mathrm{O}_{4}^{-}$ & 193.05063 & 193.05092 & -0.29 & $\begin{array}{l}\text { 134(89), } \\
\mathbf{1 4 9 ( 1 0 0 ) ,} \\
\text { 178(74) }\end{array}$ & $134(100)$ & ND \\
\hline 26 & 6.82 & $\begin{array}{l}\text { Feruloyl pento- } \\
\text { side }\end{array}$ & $\mathrm{C}_{15} \mathrm{H}_{17} \mathrm{O}_{8}^{-}$ & 325.09289 & 325.09482 & -1.93 & 193(100) & $\begin{array}{r}\text { 134(100) } \\
149(40) \\
178(10)\end{array}$ & ND \\
\hline 27 & 6.89 & $\begin{array}{l}\text { Feruloyl-isocitric } \\
\text { acid }\end{array}$ & $\mathrm{C}_{16} \mathrm{H}_{15} \mathrm{O}_{10}^{-}$ & 367.06707 & 367.06703 & 0.04 & $\begin{array}{l}\text { 111(16), 155(8), } \\
\mathbf{1 7 3}(100) \\
\text { 191(20), } \\
321(12)\end{array}$ & $\begin{array}{l}111(100), 129(9) \\
155(32)\end{array}$ & ND \\
\hline 28 & 7.62 & $\begin{array}{l}\text { Caffeoyl-feruloyl- } \\
\text { quinic acid } \\
\text { isomer } 1\end{array}$ & $\mathrm{C}_{26} \mathrm{H}_{25} \mathrm{O}_{12}^{-}$ & 529.13515 & 529.13590 & -0.75 & $\begin{array}{l}\text { 173(35), 193(6), } \\
335(20), 349(4) \\
\mathbf{3 6 7}(100)\end{array}$ & $\begin{array}{r}\text { 173(100) } \\
193(20)\end{array}$ & 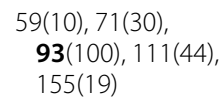 \\
\hline 29 & 7.72 & $\begin{array}{l}\text { Dihydroxyben- } \\
\text { zoyl-feruloyl } \\
\text { acid hexoside }\end{array}$ & $\mathrm{C}_{23} \mathrm{H}_{23} \mathrm{O}_{12}^{-}$ & 491.11950 & 491.11755 & 1.95 & $\begin{array}{l}\text { 161(10), } \\
\mathbf{3 1 5}(100) \\
323(30) \\
447(15) \\
459(22)\end{array}$ & $\begin{array}{l}\text { 108(13), 151(10), } \\
\text { 153(100), } \\
\text { 163(14), } \\
165(19)\end{array}$ & 109(100) \\
\hline 30 & 7.97 & $\begin{array}{l}\text { Caffeoyl-feruloyl- } \\
\text { quinic acid } \\
\text { isomer } 2\end{array}$ & $\mathrm{C}_{26} \mathrm{H}_{25} \mathrm{O}_{12}^{-}$ & 529.13515 & 529.13505 & 0.10 & $\begin{array}{l}\text { 173(10), 193(5), } \\
335(6), 353(7), \\
\mathbf{3 6 7}(100)\end{array}$ & $\begin{array}{l}\text { 134(6), 173(100), } \\
\text { 193(61) }\end{array}$ & $\begin{array}{l}\text { 71(30), 93(100), } \\
\text { 109(18), 111(54), } \\
\text { 155(20) }\end{array}$ \\
\hline \multicolumn{10}{|c|}{ Flavonoid glycosides and aglycones } \\
\hline 31 & 5.57 & $\begin{array}{l}\text { Apigenin 6,8-di- } \\
\text { C-hexoside }\end{array}$ & $\mathrm{C}_{27} \mathrm{H}_{29} \mathrm{O}_{15}^{-}$ & 593.15119 & 593.15504 & -3.85 & $\begin{array}{l}\text { 353(44), 383(23), } \\
\mathbf{4 7 3}(100) \\
503(31) \\
575(10)\end{array}$ & $\begin{array}{l}353(100), 354(6) \\
383(16)\end{array}$ & $\begin{array}{l}\text { 297(48), } 325(100) \\
326(7)\end{array}$ \\
\hline 32 & 6.56 & $\begin{array}{l}\text { Apigenin } \\
\text { 8-C-hexoside }\end{array}$ & $\mathrm{C}_{21} \mathrm{H}_{19} \mathrm{O}_{10}^{-}$ & 431.09837 & 431.09879 & -0.42 & $\begin{array}{l}311(100), \\
312(16), \\
341(18), 385(5), \\
413(4)\end{array}$ & $\begin{array}{r}283(100) \\
284(12)\end{array}$ & $\begin{array}{l}\text { 163(48), 183(50), } \\
211(30), 224(27), \\
\text { 239(100) }\end{array}$ \\
\hline
\end{tabular}


Table 1 (continued)

\begin{tabular}{|c|c|c|c|c|c|c|c|c|c|}
\hline No & $t_{R^{\prime}} \min$ & $\begin{array}{l}\text { Compound } \\
\text { name }\end{array}$ & $\begin{array}{l}\text { Molecular } \\
\text { formula, } \\
{[\mathrm{M}-\mathrm{H}]^{-}}\end{array}$ & $\begin{array}{l}\text { Calculated } \\
\text { mass, } \\
{[\mathrm{M}-\mathrm{H}]^{-}}\end{array}$ & $\begin{array}{l}\text { Exact mass, } \\
{[\mathrm{M}-\mathrm{H}]^{-}}\end{array}$ & $\Delta \mathrm{mDa}$ & $\begin{array}{l}\text { MS }{ }^{2} \text { Fragments, } \\
\text { (\% Base Peak) }\end{array}$ & $\begin{array}{l}\text { MS }^{3} \text { Fragments, } \\
\text { (\% Base Peak) }\end{array}$ & $\begin{array}{l}\text { MS }{ }^{4} \text { Fragments, } \\
\text { (\% Base Peak) }\end{array}$ \\
\hline 33 & 6.67 & $\begin{array}{l}\text { Quercetin } \\
\text { 3-O-glucoside }\end{array}$ & $\mathrm{C}_{21} \mathrm{H}_{19} \mathrm{O}^{12-}$ & 463.08820 & 463.08780 & 0.40 & $\begin{array}{l}300(14) \\
\text { 301(100), } \\
302(9)\end{array}$ & $\begin{array}{l}151(83) \\
\mathbf{1 7 9}(100) \\
255(31) \\
257(14) \\
271(44)\end{array}$ & $151(100)$ \\
\hline 34 & 6.70 & $\begin{array}{l}\text { Quercetin } \\
\text { 3-O-hexuro- } \\
\text { nide }\end{array}$ & $\mathrm{C}_{21} \mathrm{H}_{17} \mathrm{O}_{13}^{-}$ & 477.06746 & 477.06840 & -0.93 & $301(100), 302(9)$ & 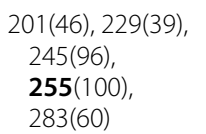 & $\begin{array}{l}\text { 155(35), 199(19), } \\
211(11) \\
\mathbf{2 2 7}(100)\end{array}$ \\
\hline 35 & 6.72 & $\begin{array}{l}\text { Scutellarein } \\
\text { 7-O-hexuro- } \\
\text { nide }\end{array}$ & $\mathrm{C}_{21} \mathrm{H}_{17} \mathrm{O}_{12}^{-}$ & 461.07255 & 461.07256 & -0.01 & $\begin{array}{l}\text { 175(3), } 285(100) \\
286(4)\end{array}$ & $\begin{array}{l}\text { 165(22), 185(23), } \\
\text { 239(54), } \\
241(34), \\
\mathbf{2 6 7}(100)\end{array}$ & $\begin{array}{l}211(8), 223(13) \\
\mathbf{2 3 9}(100)\end{array}$ \\
\hline 36 & 6.99 & $\begin{array}{l}\text { Apigenin } \\
\text { 7-O-(6"- } \\
\text { rhamnosyl)- } \\
\text { hexoside }\end{array}$ & $\mathrm{C}_{26} \mathrm{H}_{25} \mathrm{O}_{15}^{-}$ & 577.11989 & 577.12224 & -2.34 & $\begin{array}{l}269(100) \\
270(12), 307(7)\end{array}$ & $\begin{array}{l}\text { 149(35), 151(23), } \\
201(24) \\
\text { 225(100), } \\
227(21)\end{array}$ & $\begin{array}{l}\text { 169(30), 181(100), } \\
\text { 183(28), 196(20), } \\
197(63)\end{array}$ \\
\hline 37 & 7.10 & $\begin{array}{l}\text { Kaempferol } \\
\text { 3-O-glucoside }\end{array}$ & $\mathrm{C}_{21} \mathrm{H}_{19} \mathrm{O}_{11}^{-}$ & 447.09329 & 447.09209 & 1.20 & $\begin{array}{l}\text { 255(18), 284(96), } \\
\mathbf{2 8 5}(100) \\
286(14), \\
327(16)\end{array}$ & $\begin{array}{l}\text { 229(39), 241(31), } \\
\text { 256(85), } \\
\mathbf{2 5 7 ( 1 0 0 )} \\
267(45)\end{array}$ & 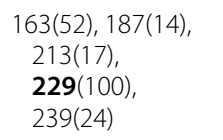 \\
\hline 38 & 7.19 & $\begin{array}{l}\text { Isorhamnetin } \\
\text { 3-O-glucoside }\end{array}$ & $\mathrm{C}_{22} \mathrm{H}_{21} \mathrm{O}_{12}^{-}$ & 477.10385 & 477.10352 & 0.33 & $\begin{array}{l}\text { 271(9), 285(11), } \\
\text { 314(100), } \\
315(31), \\
257(20)\end{array}$ & $\begin{array}{l}\text { 243(21), 271(74), } \\
\mathbf{2 8 5}(100), \\
\text { 286(42), } \\
300(16)\end{array}$ & $\mathbf{2 7 0}(100)$ \\
\hline 39 & 7.23 & $\begin{array}{l}\text { Apigenin } \\
\text { 7-O-hexoside }\end{array}$ & $\mathrm{C}_{21} \mathrm{H}_{19} \mathrm{O}_{10}{ }^{-}$ & 431.09837 & 431.09827 & 0.10 & $\begin{array}{l}\text { 268(15), } \\
\quad 269(100) \\
270(10), 311(3)\end{array}$ & $\begin{array}{l}\text { 149(33), 197(27), } \\
\text { 201(27), } \\
\text { 225(100), } \\
\text { 269(32) }\end{array}$ & $\begin{array}{l}\text { 169(31), 181(55), } \\
183(48), 196(32), \\
197(100)\end{array}$ \\
\hline 40 & 7.29 & $\begin{array}{l}\text { Apigenin } \\
\text { 7-O-hexuro- } \\
\text { nide }\end{array}$ & $\mathrm{C}_{21} \mathrm{H}_{17} \mathrm{O}_{11}{ }^{-}$ & 445.07764 & 445.07714 & 0.50 & $\begin{array}{l}\text { 175(7), 269(100) } \\
270(7)\end{array}$ & $\begin{array}{l}\text { 149(41), 151(24), } \\
\text { 201(27), } \\
\text { 225(100), } \\
227(21)\end{array}$ & $\begin{array}{l}\text { 181(100), 183(82), } \\
\text { 196(31), 197(49), } \\
210(14)\end{array}$ \\
\hline 41 & 7.45 & $\begin{array}{l}\text { Hispidulin } \\
\text { 7-O-hexuro- } \\
\text { nide }\end{array}$ & $\mathrm{C}_{22} \mathrm{H}_{19} \mathrm{O}_{12}{ }^{-}$ & 475.08820 & 475.08735 & 0.85 & $\begin{array}{l}\text { 175(6), 299(100) } \\
300(15)\end{array}$ & $\mathbf{2 8 4}(100), 285(2)$ & $\begin{array}{l}255(13), \mathbf{2 5 6}(100) \\
284(20)\end{array}$ \\
\hline 42 & 7.48 & $\begin{array}{l}\text { Jaceosidin } \\
\text { 7-0-hexoside }\end{array}$ & $\mathrm{C}_{23} \mathrm{H}_{23} \mathrm{O}_{12}^{-}$ & 491.11950 & 491.11788 & 1.62 & $\begin{array}{l}313(42), 314(40), \\
327(13), \\
329(73) \\
\mathbf{4 7 6}(100)\end{array}$ & $\begin{array}{l}313(100), \\
314(30), \\
315(12), 327(5), \\
343(17)\end{array}$ & $\begin{array}{l}\text { 282(18), 285(84), } \\
\text { 295(7), 298(100), } \\
\text { 299(10) }\end{array}$ \\
\hline 43 & 7.72 & $\begin{array}{l}\text { Kaempferide } \\
\text { 3-O-hexuro- } \\
\text { nide }\end{array}$ & $\mathrm{C}_{22} \mathrm{H}_{19} \mathrm{O}_{12}^{-}$ & 475.08820 & 475.08846 & -0.26 & $\begin{array}{l}\text { 175(13), } \\
\mathbf{2 9 9}(100) \\
300(14)\end{array}$ & 284(100) & $\begin{array}{l}227(12), \mathbf{2 5 5}(100) \\
256(17)\end{array}$ \\
\hline 44 & 7.80 & $\begin{array}{l}\text { Kaempferol } \\
\text { 3-O-acetyl- } \\
\text { hexoside }\end{array}$ & $\mathrm{C}_{23} \mathrm{H}_{21} \mathrm{O}_{12}^{-}$ & 489.10385 & 489.10605 & -2.20 & $\begin{array}{c}\text { 255(37), } \\
\mathbf{2 8 4}(100), \\
285(78), \\
286(12) \\
327(20)\end{array}$ & $\begin{array}{l}227(14) \\
255(100) \\
256(21)\end{array}$ & $\begin{array}{l}211(67), \mathbf{2 2 7}(100) \\
255(10)\end{array}$ \\
\hline 45 & 8.10 & Scutellarein & $\mathrm{C}_{15} \mathrm{H}_{9} \mathrm{O}_{6}^{-}$ & 285.04046 & 285.04116 & -0.70 & $\begin{array}{l}\text { 165(28), 223(33), } \\
239(63) \\
\mathbf{2 6 7}(100) \\
268(19)\end{array}$ & $\begin{array}{l}211(7), 223(33), \\
225(7) \\
\mathbf{2 3 9}(100)\end{array}$ & $\begin{array}{l}\text { 167(7), 183(7), } \\
\text { 195(57), } \\
\mathbf{2 1 1}(100)\end{array}$ \\
\hline 46 & 8.68 & Luteolin $^{a}$ & $\mathrm{C}_{15} \mathrm{H}_{9} \mathrm{O}_{6}^{-}$ & 285.04046 & 285.04019 & 0.27 & $\begin{array}{l}\text { 151(33), 175(80), } \\
\text { 199(77), } \\
217(64), \\
\mathbf{2 4 1 ( 1 0 0 )}\end{array}$ & $\begin{array}{l}\text { 197(91), } \\
\text { 198(100), } \\
\text { 199(87), } \\
212(16), \\
213(56)\end{array}$ & 169(44), 170(100) \\
\hline
\end{tabular}


Table 1 (continued)

\begin{tabular}{|c|c|c|c|c|c|c|c|c|c|}
\hline No & $t_{R^{\prime}} \min$ & $\begin{array}{l}\text { Compound } \\
\text { name }\end{array}$ & $\begin{array}{l}\text { Molecular } \\
\text { formula, } \\
{[\mathrm{M}-\mathrm{H}]^{-}}\end{array}$ & $\begin{array}{l}\text { Calculated } \\
\text { mass, } \\
{[\mathrm{M}-\mathrm{H}]^{-}}\end{array}$ & $\begin{array}{l}\text { Exact mass, } \\
{[\mathrm{M}-\mathrm{H}]^{-}}\end{array}$ & $\Delta \mathrm{mDa}$ & $\begin{array}{l}\text { MS }^{2} \text { Fragments, } \\
\text { (\% Base Peak) }\end{array}$ & $\begin{array}{l}\text { MS }^{3} \text { Fragments, } \\
\text { (\% Base Peak) }\end{array}$ & $\begin{array}{l}\text { MS }{ }^{4} \text { Fragments, } \\
\text { (\% Base Peak) }\end{array}$ \\
\hline 47 & 8.81 & Nepetin & $\mathrm{C}_{16} \mathrm{H}_{11} \mathrm{O}_{7}^{-}$ & 315.05103 & 315.05103 & 0.00 & $\begin{array}{r}300(100) \\
301(11)\end{array}$ & $\begin{array}{c}216(100) \\
227(78) \\
228(88) \\
255(48) \\
272(67)\end{array}$ & $\begin{array}{l}\text { 173(21), 187(8), } \\
\mathbf{1 8 8}(100) \\
\text { 201(25) }\end{array}$ \\
\hline 48 & 9.54 & Apigenin $^{a}$ & $\mathrm{C}_{15} \mathrm{H}_{9} \mathrm{O}_{5}^{-}$ & 269.04555 & 269.04539 & 0.16 & 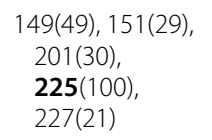 & $\begin{array}{l}\text { 169(22), 180(20), } \\
\mathbf{1 8 1}(100) \\
\text { 183(36), } \\
197(37)\end{array}$ & ND \\
\hline 49 & 9.70 & Kaempferol $^{a}$ & $\mathrm{C}_{15} \mathrm{H}_{9} \mathrm{O}_{6}^{-}$ & 285.04046 & 285.04052 & -0.06 & $\begin{array}{l}\text { 183(71), 211(98), } \\
212(10), 213(8) \\
\mathbf{2 2 7}(100)\end{array}$ & $\begin{array}{r}\mathbf{1 8 3}(100) \\
199(56) \\
250(17)\end{array}$ & ND \\
\hline 50 & 9.72 & Chrysoeriol $^{a}$ & $\mathrm{C}_{16} \mathrm{H}_{11} \mathrm{O}_{6}^{-}$ & 299.05611 & 299.05580 & 0.31 & $\begin{array}{r}\mathbf{2 8 4}(100) \\
285(11)\end{array}$ & $\begin{array}{l}\text { 137(68), } 212(58), \\
\mathbf{2 2 7}(100) \\
228(75), \\
255(36)\end{array}$ & $\begin{array}{c}\text { 183(100), } 199(20) \\
200(17), 213(5)\end{array}$ \\
\hline 51 & 9.74 & Jaceidin & $\mathrm{C}_{18} \mathrm{H}_{15} \mathrm{O}_{8}^{-}$ & 359.07724 & 359.07734 & -0.10 & $\begin{array}{r}\mathbf{3 4 4}(100) \\
345(15)\end{array}$ & $329(100)$ & $\begin{array}{l}242(12), 270(14), \\
286(6), 301(73), \\
\mathbf{3 1 4}(100)\end{array}$ \\
\hline 52 & 9.97 & Jaceosidin & $\mathrm{C}_{17} \mathrm{H}_{13} \mathrm{O}_{7}^{-}$ & 329.06668 & 329.06647 & 0.21 & $\begin{array}{r}\text { 314(100), } \\
315(11)\end{array}$ & $\mathbf{2 9 9}(100), 300(3)$ & $\begin{array}{l}\text { 199(6), 227(9), } \\
243(4), 255(11) \\
\mathbf{2 7 1}(100)\end{array}$ \\
\hline 53 & 10.03 & Kaempferide & $\mathrm{C}_{16} \mathrm{H}_{11} \mathrm{O}_{6}^{-}$ & 299.05611 & 299.05637 & -0.25 & $\begin{array}{r}\mathbf{2 8 4}(100) \\
285(12)\end{array}$ & $\begin{array}{l}227(7), 255(100) \\
256(8)\end{array}$ & $\mathrm{ND}$ \\
\hline 54 & 11.20 & Eupatorin & $\mathrm{C}_{18} \mathrm{H}_{15} \mathrm{O}_{7}^{-}$ & 343.08233 & 343.08217 & 0.16 & $\begin{array}{r}328(100) \\
329(10)\end{array}$ & $\mathbf{3 1 3}(100)$ & $\begin{array}{l}270(10), 282(43), \\
283(10), 285(43), \\
\mathbf{2 9 8}(100)\end{array}$ \\
\hline 55 & 11.24 & Centaureidin & $\mathrm{C}_{18} \mathrm{H}_{15} \mathrm{O}_{8}^{-}$ & 359.07724 & 359.07681 & 0.43 & $\begin{array}{l}301(10) \\
\mathbf{3 4 4}(100) \\
345(12)\end{array}$ & $\begin{array}{r}301(100) \\
329(36)\end{array}$ & $\begin{array}{l}\text { 258(6), 270(9), } \\
\mathbf{2 8 6}(100)\end{array}$ \\
\hline
\end{tabular}

a Confirmed using standards, the other compounds were identified according HRMS data and MSn; tR - retention time (min); $\Delta$ mDa - mean mass accuracy; "ND" not detected

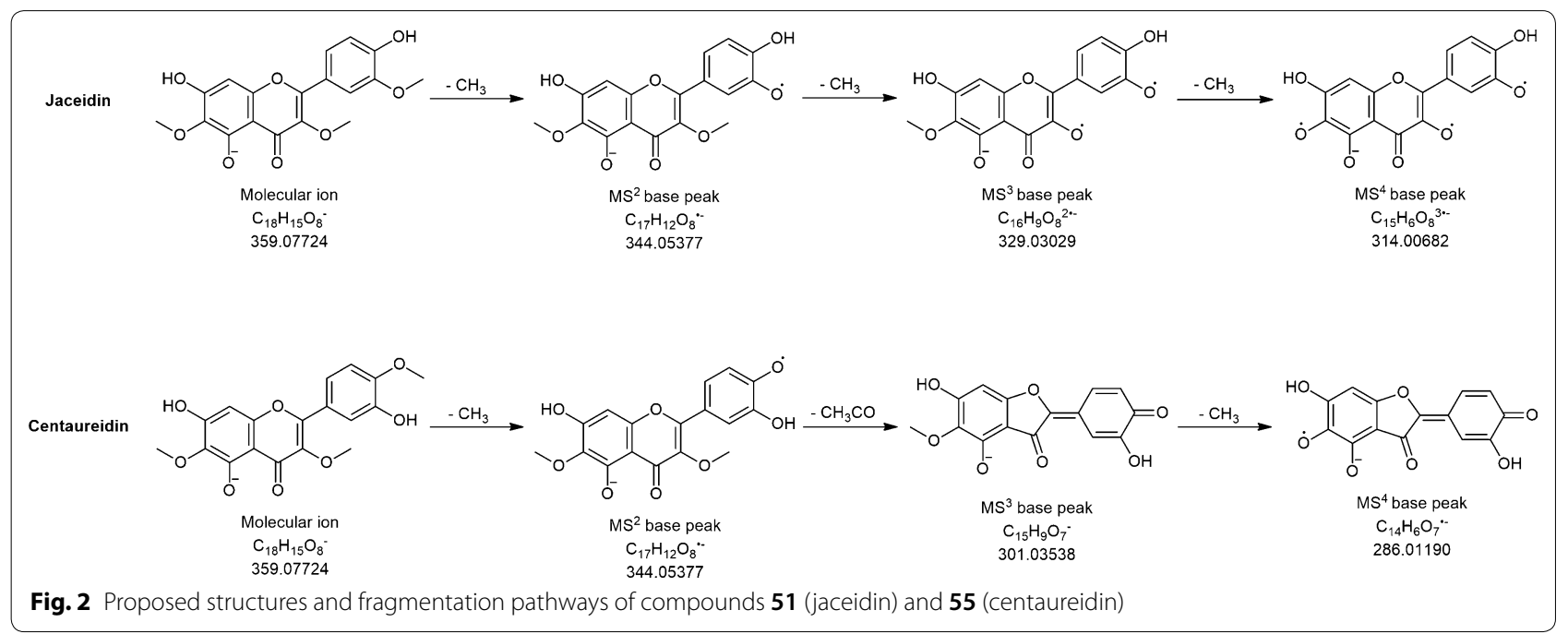


in $\mathrm{MeOH}$ and $\mathrm{EtOH}$ extract, the highest amount of gallic acid, as well as gentisic acid, was recorded. The highest concentrations of flavonoid aglycones and glycosides were observed in $\mathrm{Me}_{2} \mathrm{CO}$ extract. Further, quercetin 3-O-glucoside, isorhamnetin 3-O-glucoside and kaempferol were not detected in EtOAc extract.

Due to a lack of standard compounds, the presence of compounds can be expressed in each extract through the $\mathrm{m} / \mathrm{z}$ peak area depending of their intensities (Additional file 1: Table S2). Higher intensity of eupatorin, nepetin and jaceosidin were observed in all extracts, still their intensities were 1.12-2.36 times higher in EtOAc extract. Jaceidin, jaceosidin 7-O-hexoside and centaureidin were with the highest intensity in EtOAc extract, whereas intensity of jaceidin was 2.6-8.2 times higher than in all other extracts, especially comparing to the $\mathrm{MeOH}$ and $\mathrm{Me}_{2} \mathrm{CO}$ extract. The highest intensity of kaempferide and scutellarein were observed in $\mathrm{Me}_{2} \mathrm{CO}$ extract, while among other extracts they were least represented in EtOAc extract. Two feruloyl-quinic acid isomers were among the most represented compounds in all extracts, with the exception of EtOAc extract.

\section{Principal component analysis}

Principal component analysis was applied to classify 55 plant metabolites from five extracts obtained using different solvents based on solvent polarity (Fig. 3). The PC1 describes $83.09 \%$, while PC2 accounted for $11.80 \%$ of the total variability. Two-dimensional PCs showed a separation between three groups of metabolites: flavonoids such as $\mathbf{4 7}, \mathbf{4 8}, \mathbf{5 0}, \mathbf{5 2}$, and $\mathbf{5 4}$ formed one cluster, phenolic acid derivatives such as $\mathbf{2 , 1 1}, \mathbf{1 6}, \mathbf{2 2}, 37$ formed the second group of samples, while other metabolites third group. EtOAc and DCM: $\mathrm{MeOH}$ has the most influence on the first group of metabolites (flavonoids), while alcoholic solvents as more polar solvent showed influence on the second group of metabolites (phenolic acid derivatives). Acetone solvent showed influence on both groups of metabolites. Aforementioned results are in agreement with Additional file 1: Table S2, whereas metabolites 47, 48, 50, 52 and 54 were extracted in the highest amount by ethyl acetate and acetone, while metabolites such as $\mathbf{2}$, 11, 16, 22, and 37 were extracted by alcoholic solvents and acetone. Other metabolites do not show any significant difference between five solvents. To classify metabolite profiles between five different extracts, PCA was applied: two novel PCs describing the maximum variation among the data were found. PC1 and PC2 accounted for $55.93 \%$ and $26.16 \%$, of the total variance. Based on Fig. 3 it can be concluded that ethyl-acetate extract differs from all other extracts, while $\mathrm{EtOH}, \mathrm{MeOH}$ and $\mathrm{Me}_{2} \mathrm{CO}$ were more similar (Fig. 3). According to the loading plot (Figs. 3d), the most important metabolites discriminating between solvents are $\mathbf{4 2}, \mathbf{4 7}, \mathbf{5 1}, \mathbf{5 4}$, and $\mathbf{5 5}$. PC2 showed a negative loading value of metabolites $2,12,17$, and 25 , while metabolites such as $\mathbf{3 2 , 5 0}$ and $\mathbf{5 3}$ were positively related to $\mathrm{PC} 2$.

\section{Antimicrobial activity Well diffusion}

Well diffusion was used as an initial screening for the antimicrobial activity of the $C$. calcitrapa plant extracts against fourteen bacterial strains and yeast $C$. albicans. According to the obtained results (Table 2), all tested extracts, exhibited an antimicrobial effect. C. albicans, $X$. a. pv. juglandis 320 and 321 were resistant to the all extracts at the tested concentrations. Phytopathogenic isolates of E. amylovora, $P$. s. pv. syringae and $X . c$. pv. campestris were among the most sensitive strains to all tested extracts. Among human pathogens, the best inhibitory effect of extracts was recorded against MRSA. Also some of the isolates showed a moderate sensitivity to all extracts, while others had a high degree of sensitivity to the particular extract. The growth of MRSA and S. aureus was significantly inhibited by $\mathrm{MeOH}$ and EtOAc extracts, while the remaining extracts appeared to be moderately inhibitory. The EtOAc extract had a complete inhibitory effect on the growth of $E$. coli, while the remaining extracts caused sparse growth of this pathogen. Complete growth inhibition was observed towards strains $P$. $s$. pv. aptata P29 and P49, with the lowest activity observed for EtOH extract. A strong antimicrobial potential was recorded against E. amylovora, $X . c$. pv. campestris and $P$. s. pv. syringae for all of the extracts. The DCM: $\mathrm{MeOH}$ extract had a moderately inhibitory effect against $A$. tumefaciens, while the remaining extracts showed a high degree of growth inhibition. The best inhibitory effect against all tested strains showed EtOAc extract, with the highest zones of inhibition against $P$. s. pv. syringae $(30.5 \mathrm{~mm}), X$. c. pv. campestris $(30 \mathrm{~mm})$ and A. tumefaciens $(28.5 \mathrm{~mm})$. C. albicans, E. faecalis, L. monocytogenes, S. aureus and MRSA were sensitive to tested antibiotic/mycotic, while the remaining bacterial strains showed a resistance.

\section{MIC assay}

After the initial screening, all extracts of C. calcitrapa were used in MIC assay against the most susceptible strains from the previous assay (Table 2). Among the strains used in this assay, A. tumefaciens and E. coli were more resistant. The MIC values for $A$. tumefaciens were in the range of $250-750 \mu \mathrm{g} / \mathrm{mL}$ depending on the extract, and for $E$. coli those values were similar, with exception for EtOH extract, without growth inhibition scored at the maximal tested concentration (Table 3). All extracts inhibited the growth of MRSA at lower concentrations 

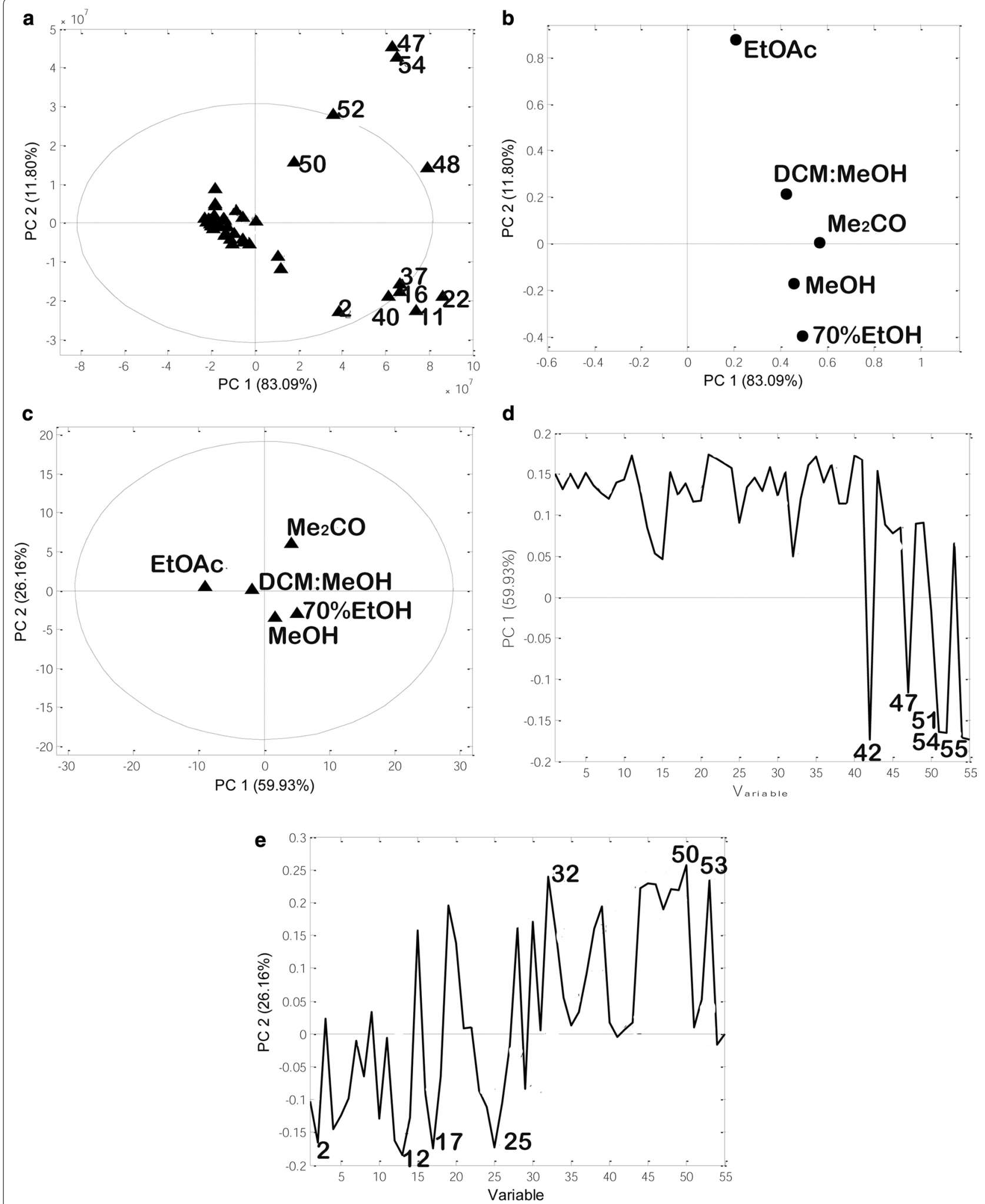

Fig. 3 PCA analysis of tested extracts based on peak area: a PCs score plot, $\mathbf{b}$ loading plot, c PCs score plot based on extraction solvents, $\mathbf{d}$ loading plot PC1 and e loading plot PC2 
Table 2 Well diffusion assay of different C. calcitrapa extracts

\begin{tabular}{|c|c|c|c|c|c|c|}
\hline Tested microorganisms & $\mathrm{MeOH}$ & EtOH & EtOAC & $\mathrm{Me}_{2} \mathrm{CO}$ & DCM:MeOH & $\begin{array}{l}\text { Antibiotic/ } \\
\text { Mycotic }\end{array}$ \\
\hline Candida albicans ATCC 10231 & - & - & - & - & - & $13 \pm 1.15$ \\
\hline Enterococcus faecalis ATCC 29212 & - & - & $13 \pm 0.29$ & $9 \pm 0.00$ & - & $13.25 \pm 0.14$ \\
\hline Escherichia coli ATCC 25922 & $15.5 \pm 0.29$ & $12.25 \pm 0.14$ & $14 \pm 0.00$ & $12.5 \pm 0.29$ & $13 \pm 0.00$ & - \\
\hline Listeria monocytogenes & $13.17 \pm 0.17$ & - & $18.5 \pm 0.29$ & $9.25 \pm 0.14$ & - & $17 \pm 0.00$ \\
\hline Methicillin resistant S. aureus ATCC 33591 & $21 \pm 0.00$ & $12.5 \pm 0.29$ & $20 \pm 0.00$ & $14 \pm 0.58$ & $12.5 \pm 0.29$ & $16 \pm 1.15$ \\
\hline Staphylococcus aureus ATCC 25923 & $16.17 \pm 0.17$ & $13.5 \pm 0.29$ & $21.17 \pm 0.17$ & $12 \pm 0.29$ & $13.5 \pm 0.29$ & $16.75 \pm 0.43$ \\
\hline Agrobacterium tumefaciens & $21.83 \pm 0.17$ & $21 \pm 0.29$ & $28.5 \pm 0.29$ & $16.17 \pm 0.17$ & $14 \pm 0.00$ & - \\
\hline Erwinia amylovora NCPPB 683 & $24.5 \pm 0.29$ & $20.5 \pm 0.87$ & $26.5 \pm 1.44$ & $20.5 \pm 0.29$ & $23 \pm 0.29$ & - \\
\hline Pseudomonas syringae pv. aptata P16 & $10.67 \pm 0.33$ & $9.83 \pm 0.17$ & $10.75 \pm 1.01$ & $11.33 \pm 0.44$ & $12.5 \pm 0.87$ & - \\
\hline Pseudomonas syringae pv. aptata P29 & $16 \pm 0.58$ & $14 \pm 0.58$ & $24.75 \pm 0.14$ & $19 \pm 0.00$ & $20 \pm 0.58$ & - \\
\hline Pseudomonas syringae pv. aptata P49 & $13.75 \pm 0.43$ & $11.5 \pm 0.29$ & $21 \pm 0.00$ & $12.5 \pm 0.00$ & $16.5 \pm 0.29$ & - \\
\hline Pseudomonas syringae pv. syringae GSPB 1142 & $19.75 \pm 0.14$ & $21 \pm 0.00$ & $30.5 \pm 0.29$ & $24.25 \pm 0.43$ & $27 \pm 0.00$ & - \\
\hline Xanthomonas arboricola pv. juglandis 320 & - & - & - & - & - & - \\
\hline Xanthomonas arboricola pv. juglandis 321 & - & - & - & - & - & - \\
\hline Xanthomonas campestris pv. campestris NCPPB 583 & $27 \pm 0.00$ & $22 \pm 0.00$ & $30 \pm 0.00$ & $27.25 \pm 0.14$ & $25.25 \pm 0.14$ & - \\
\hline
\end{tabular}

Antibiotic - vancomycin; Mycotic — nystatin; - no activity. The growth inhibition zones are expressed in mm and represented as mean

values of three independent replicates $\pm \mathrm{SE}$

Table 3 Minimum inhibitory (MIC) and minimum bactericidal concentration (MBC) of C. calcitrapa extracts

\begin{tabular}{|c|c|c|c|c|c|c|c|}
\hline Bacterial strains & $\begin{array}{l}\mathrm{MeOH} \\
\mathrm{MIC}\end{array}$ & EtOH & EtOAc & $\mathrm{Me}_{2} \mathrm{CO}$ & DCM:MeOH & Str & Van \\
\hline E. coli ATCC 25922 & 375 & - & 200 & 375 & 375 & 4.6 & 500 \\
\hline MRSA ATCC 33591 & 15 & 60 & 15 & 7.8 & 31 & - & - \\
\hline S. aureus ATCC 25923 & 94 & 125 & 16 & 31 & 62.5 & 9 & 1.6 \\
\hline A. tumefaciens & 500 & 750 & 250 & 375 & 250 & - & - \\
\hline E. amylovora NCPPB 683 & 200 & 375 & 13 & 375 & 150 & 3 & 400 \\
\hline P. syringae pv. aptata P29 & 250 & 500 & 100 & 500 & 250 & 1.5 & - \\
\hline P. syringae pv. syringae GSPB 1142 & 200 & 200 & 13 & 150 & 100 & 3 & - \\
\hline \multirow[t]{2}{*}{ X. campestris pv. campestris NCPPB 583} & 200 & 500 & 25 & 150 & 25 & 12.5 & 12.5 \\
\hline & $\mathrm{MBC}$ & & & & & & \\
\hline E. coli ATCC 25922 & 500 & - & 250 & 500 & 500 & 6 & 100 \\
\hline MRSA ATCC 33591 & 1000 & - & 1000 & 500 & 500 & - & - \\
\hline S. aureus ATCC 25923 & 125 & 250 & 125 & 125 & 250 & 25 & 3 \\
\hline A. tumefaciens & 1000 & 1000 & 500 & 1000 & 1000 & - & - \\
\hline E. amylovora NCPPB 683 & 500 & 500 & 25 & 500 & 200 & 6 & - \\
\hline P. syringae pv. aptata P29 & - & - & 500 & - & 500 & 6 & - \\
\hline P. syringae pv. syringae GSPB 1142 & 500 & 500 & 25 & 200 & 200 & 6 & - \\
\hline X. campestris pv. campestris NCPPB 583 & 500 & - & 150 & 500 & 500 & 25 & 25 \\
\hline
\end{tabular}

Str-streptomycin; Van-vancomycin. (-) - not detected in the range of tested concentrations. Values are expressed in $\mu \mathrm{g} / \mathrm{mL}$

than $S$. aureus, while MBC were much higher than $S$. aureus. MRSA proved to be the most sensitive isolate to all tested extracts. The best inhibitory effect against $P$. $s$. pv. syringae and $P$. s. pv. aptata showed EtOAc extract (MIC values of $31 \mu \mathrm{g} / \mathrm{mL}$ and $100 \mu \mathrm{g} / \mathrm{mL}$, respectively). Differences are also evident in the bactericidal activity of the extracts against both strains, with $P . s$. pv. syringae, as a more susceptible strain. The MIC values of the EtOAc extract against $E$. amylovora and $X . c$. pv. campestris were 13 and $25 \mu \mathrm{g} / \mathrm{mL}$, respectively. Also, DCM:MeOH extract showed strong activity against $X . c$. pv. campestris at $25 \mu \mathrm{g} / \mathrm{mL}$. According to the results (Table 3), 
the best antimicrobial effect was recorded for EtOAc extract against all tested strains, which was in accordance with the results of the diffusion test. Based on the average MIC values towards all strain tested (Fig. 4), the antimicrobial activity of the C. calcitrapa extracts can be presented in following order: EtOAc $>$ DCM: $\mathrm{MeOH}>\mathrm{MeOH}>\mathrm{Me}_{2} \mathrm{CO}>\mathrm{EtOH}$.

\section{Cytotoxicity}

The results of cytotoxic activity of tested extracts were shown in Fig. 5. The viability of MRC-5 cells exposed to

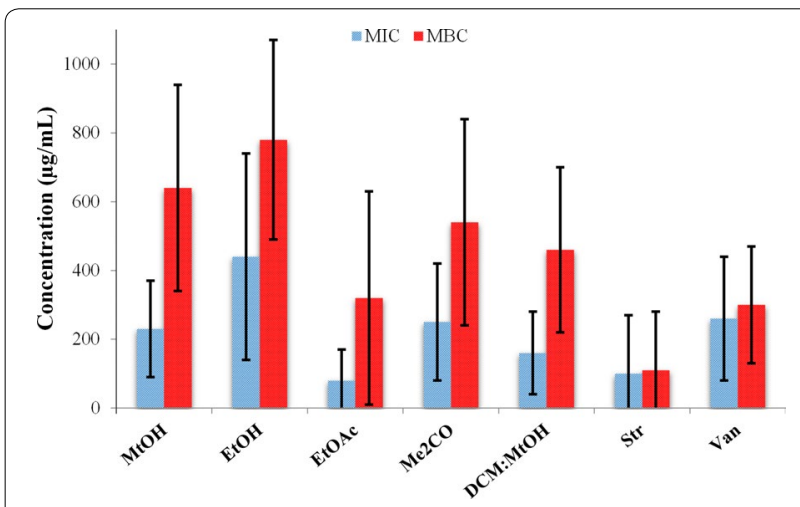

Fig. 4 Comparison of MIC and MBC average values of the extracts towards all tested strains
C. calcitrapa extracts showed lowest statistically insignificant cytotoxicity up to concentration of $62.5 \mu \mathrm{g} / \mathrm{mL}$. The EtOAc extract at 31.25 and $62.5 \mu \mathrm{g} / \mathrm{mL}$, showed slightly statistically significant elevated cytotoxicity than other extracts, with the reduction between 27 and 35\% of the cell viability, respectively. On the other hand, $\mathrm{MeOH}$, $\mathrm{EtOH}$ and DCM:MeOH extracts at concentration of $125 \mu \mathrm{g} / \mathrm{mL}$ showed statistically insignificant and equal cytotoxicity, while only EtOH extract at highest applied concentration $(250 \mu \mathrm{g} / \mathrm{mL})$ was exhibited a lowest cytotoxic effect. This finding also has been confirmed by calculated and predicted relative $\mathrm{IC}_{50}$ value of $1578 \mu \mathrm{g} / \mathrm{mL}$. The $\mathrm{IC}_{50}$ absolute and relative values of the other extracts were also calculated (Fig. 6). The highest cytotoxicity was recorded for EtOAc and $\mathrm{Me}_{2} \mathrm{CO}$ extracts with the lowest relative and absolute $\mathrm{IC}_{50}$ values between 88 and $102 \mu \mathrm{g} / \mathrm{mL}$. Moderate cytotoxicity was established for the $\mathrm{MeOH}$ and DCM: $\mathrm{MeOH}$ extracts with relative $\mathrm{IC}_{50}$ values at 158 and $147 \mu \mathrm{g} / \mathrm{mL}$, respectively.

Based on a comparative analysis of the MIC and MTT test results, at concentrations considered non-toxic, the growth of methicillin-resistant $S$. aureus was successfully inhibited by all tested extracts. Also, non-toxic concentrations of EtOAc and $\mathrm{Me}_{2} \mathrm{CO}$ extract successfully affected the growth of MRSA and S. aureus at very low concentrations (Table 3). Low non-toxic concentrations of EtOAc and DCM: $\mathrm{MeOH}$ extracts were successful against $X . c$. pv. campestris, while for E. amylovora and $P$.

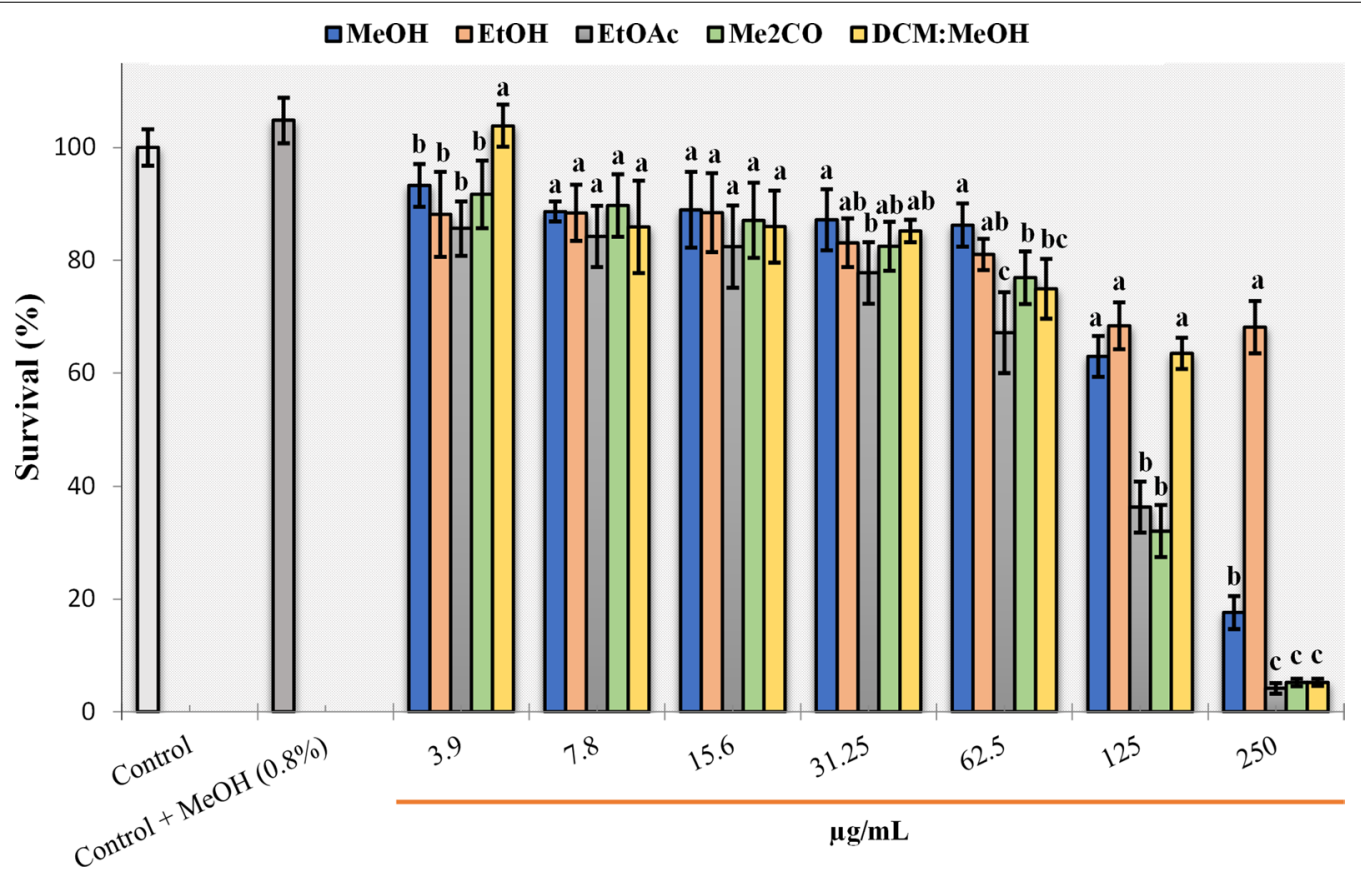

Fig. 5 Survival of MRC-5 cells in the treatment with C. calcitrapa extracts. Values followed by the same letter on the histogram columns with different concentrations were not significantly different $(P<0.05)$ according to Tukey's HSD test 

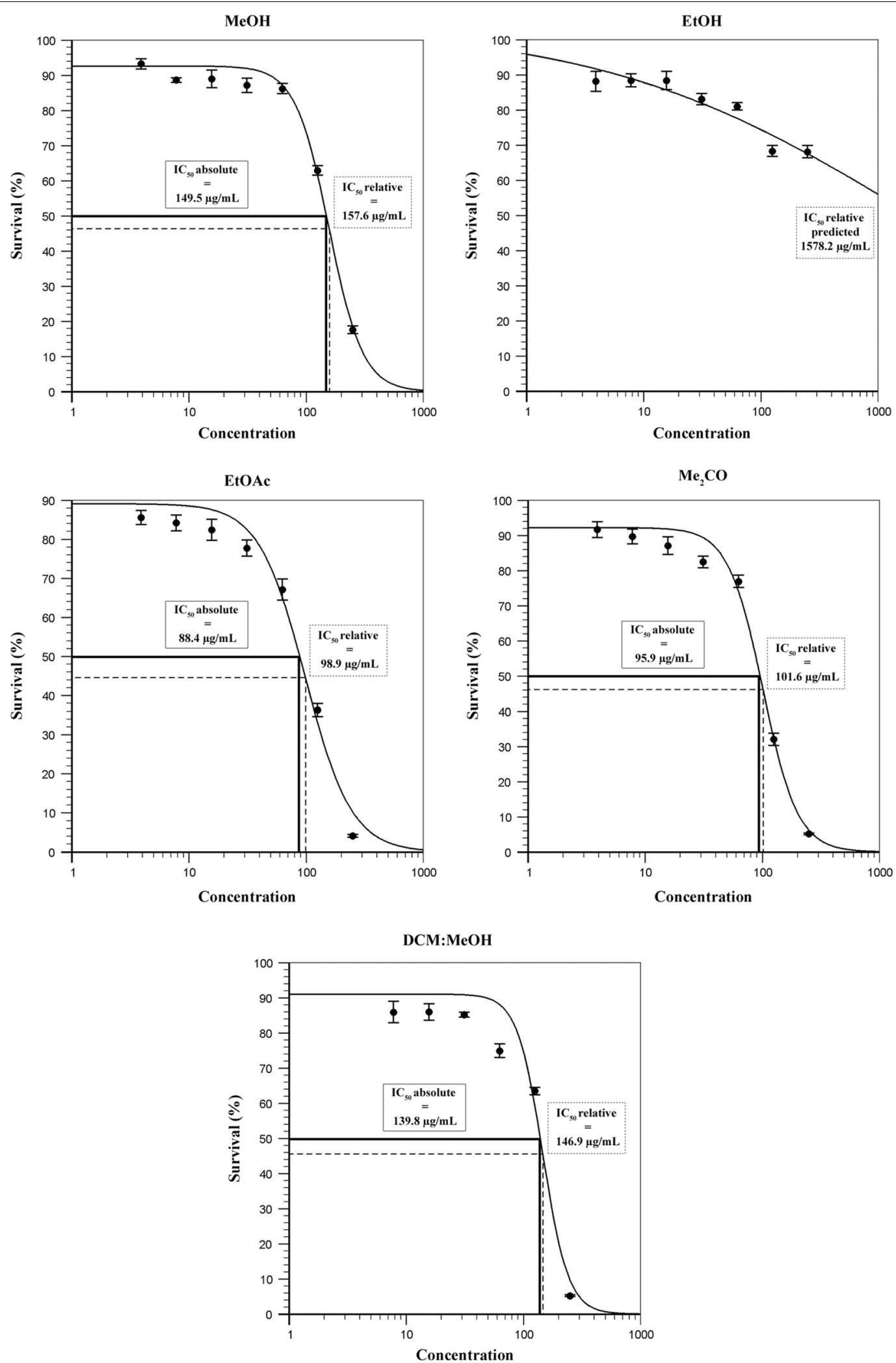

Fig. 6 The $\mathrm{IC}_{50}$ absolute and relative values of the C. calcitrapa extracts 
s. pv. syringae low non-toxic MICs were obtained only for EtOAc extract.

\section{Discussion}

Resulting extracts within this study, obtained using mixtures of water and solvent had a higher yield than the extracts obtained using pure solvents itself. It can be seen that extraction yield increases with increasing polarity of the solvent used in extraction. As EtOH and $\mathrm{Me}_{2} \mathrm{CO}$ were made as water-solvent mixtures, others compounds, such as sugars and organic acids, could be co-extracted and influenced the highest yield of these solvents. Karamenderes et al. (2007) examined the $n$-hexane, Chl (chloroform) and $\mathrm{MeOH}$ extracts of aerial parts of several Centaurea species and reported that the highest yield was obtained using $\mathrm{MeOH}$ as a solvent, which was the case in our work as well. Also, it can be spotted that $\mathrm{Me}_{2} \mathrm{CO}$ solvent mostly influenced the extraction of hydroxylated flavonoids, while EtOAc was the most efficient solvent for extraction of methoxylated flavonoids. The differences in the efficiency of the used solvents were also visible in the extraction of phenolic acids. More polar hydroxybenzoic acids were most extracted with $\mathrm{EtOH}$ and $\mathrm{MeOH}$ solvents. Still, all solvents used similarly influenced the extraction of protocatechuic acid, with exception of EtOAc which was the least efficient solvent. $\mathrm{Me}_{2} \mathrm{CO}$ and DCM: $\mathrm{MeOH}$ were the most efficient for extraction of $p$-hydroxybenzoic acid with similar extraction capacity. $\mathrm{Me}_{2} \mathrm{CO}$ solvent had the greatest influence on the extraction of hydroxycinnamic acids, $p$-coumaric and caffeic acid. Compared to the other solvents used, $\mathrm{MeOH}$ was proved to be the best solvent for the extraction of ferulic acid. However, water-ethanol mixture gave the most satisfactory results when it comes to extraction of chlorogenic acid. Methodology applied through using UHPLC-LTQ Orbitrap MS reveals 55 plant metabolites and fragmentation data confirmed the presence of many compounds which are revealed for the first time in C. calcitrapa extracts such as jaceidin and its positional isomer centaureidin. MS fragmentation data about fragmentation of jaceidin and its isomer were consistent with previously published MS data (Taamalli et al. 2015). Among the quantified flavonoids, apigenin was the most abundant in our study, which has also been identified in other Calcitrapa species such as C. pallescens Delile, $C$. urvillei, C. affinis Friv., C. cyanus, C. chilensis Bertero ex Hook. \& Arn., C. nigra L., C. virgata Lam. (Khammar and Djeddi 2012). Many phenolics identified in C. calcitrapa extracts, have been isolated from numerous Centaurea species (Erel et al. 2011; Khammar and Djeddi 2012; Azzouzi et al. 2016). However, the chemical composition was dissimilar to this study to a certain extent. Therefore, C. calcitrapa can be considered as a species with high phenolic diversity. Flavonoids such as apigenin, luteolin, kaempferol, kaempferol 3-O-glucoside, eupatorin and jaceosidin have been previously detected in the C. calcitrapa (Al-Easa and Rizk 1992; Formisano et al. 2012; Kitouni et al. 2015). In the study of Öksüz et al. (1984), apigenin and jaceosidin have been isolated from all of the investigated Centaurea species (C. virgata, $C$. kilea Boiss., C. inermiss Velen.), whereas apigenin was the major isolated compound. Also, eupatorin, nepetin and kaempferol 3-O-glucoside found to be constituents of $C$. virgata and $C$. inermis. However, eupatorin and jaceosidin besides the above mentioned species have been found together in aerial parts of C. grisebatchii (Nyman) Heldr (Djeddi et al. 2008a). Nepetin and protocatechuic acid from $C$. calcitrapa leaves have been previously detected in the related species C. melitensis L. (Ayad et al. 2012; Khammar and Djeddi 2012). Chlorogenic acid together with protocatechuic acid, kaempferol 3-O-glucoside and quercetin 3-O-glucoside has been obtained from $C$. isaurica Hub.-Mor extracts (Flamini et al. 2004). Combination of apigenin, luteolin, kaempferol, chlorogenic acid and caffeic acid as natural compounds has also been found in aerial parts of C. cyanus (Litvinenko and Bubenchikova 1988). Also, nepetin, luteolin, centaureidin, jaceidin, caffeic acid and protocatechuic acid have been isolated together from C. bracteata (Flamini et al. 2001).

A strong inhibitory effect of EtOAc extract of numerous Centaurea species, except for C. calcitrapa, has been reported, so far, by using disk diffusion method, against many bacteria, including $S$. aureus, $E$. coli and $L$. monocytogenes (Güven et al. 2005). The inhibitory activity of $\mathrm{MeOH}$ extract of $C$. calcitrapa leaves against $S$. aureus $(18 \mathrm{~mm})$ and $E$. coli $(20 \mathrm{~mm})$ has been reported in Moghannem et al. (2016), which confirmed by the results obtained in our study. Also, activity of $70 \% \mathrm{MeOH}$ extract of C. calcitrapa aerial parts $(100 \mathrm{mg} / \mathrm{mL})$ has been reported by disk diffusion method towards $S$. aureus and $E$. coli, whereas extract found to be inactive against $E$. coli at the tested concentration and moderately active against S. aureus with $13 \mathrm{~mm}$ in diameter (Soumia et al. 2014). Furthermore, Güven et al. (2005) investigated antimicrobial potential of EtOAc, Chl, $\mathrm{Me}_{2} \mathrm{CO}$ and $\mathrm{EtOH}$ extracts of several Centaurea species, except for C. calcitrapa, where EtOAc extract showed the best activity against tested microorganisms and was further examined with the microdilution method. Obtained MIC values against S. aureus at a concentration of $125 \mu \mathrm{g} / \mathrm{mL}$ for C. ptosomipappoides Wagenitz, C. odyssei Wagenitz, C. ptosomipappa Hayek, C. kurdica Reichardt, and $62.5 \mu \mathrm{g} / \mathrm{mL}$ for C. amonicola Hub. Mon., was higher than in our study. Antimicrobial activity of $\mathrm{MeOH}$ extract of capitula (diffusion method) of C. calcitrapa has been reported in the literature, with an inhibitory effect against certain bacterial 
strains including the same strains of $S$. aureus and $E$. coli as in this study (Toribio et al. 2004). While extract inhibited growth of S. aureus at concentration of $400 \mu \mathrm{g} / \mathrm{mL}$, it was reported as inactive towards $E$. coli at the maximum concentration tested. Comparing our results with the literature data, it was evident that $\mathrm{MeOH}$ extract of $\mathrm{C}$. calcitrapa leaves exhibits significantly higher antimicrobial activity, at lower concentrations, than the $\mathrm{MeOH}$ extracts of the capitula and $70 \% \mathrm{MeOH}$ extract of the entire aerial parts of this species.

Antimicrobial activity of phenolics has been quite investigated on different bacterial strains. There are few reports about the structure-activity relationship of phenolics against MRSA and E.coli. Xu and Lee (2001) have been investigated flavonoids with different substitutions for their activity towards antibiotic-resistant bacteria and found that flavonols or flavones with more hydroxyl groups on the $\mathrm{A}$ and $\mathrm{B}$ rings, are better inhibitors of MRSA, whereas methoxyl substitutions reduce activity. Furthermore, they reported that a $-\mathrm{OH}$ at $\mathrm{C} 3$ is required for anti-MRSA activity of flavonols. Also, Alcaraz et al. (2000) highlighted that 5-hydroxylation of flavones is the one of greatest importance for their activity. All flavones found in C. calcitrapa extracts had a $-\mathrm{OH}$ at $\mathrm{C} 5$ position and were tri- or tetrahydroxyflavones. Furthermore, apigenin, scutellarein, luteolin and kaempferol, which are polyhydroxylated and without methoxy groups, may be responsible for the best activity of $\mathrm{Me}_{2} \mathrm{CO}$ extract towards MRSA, due to their highest intensities in this extract. In favor of this statement is the fact, from our study, that intensity of scutellarein was observed far more in $\mathrm{Me}_{2} \mathrm{CO}$ extract than in EtOAc extract, more precisely 169 times higher. Apigenin and luteolin have been reported for strong activity against the same MRSA strain used in this study, and while luteolin was active at $62.5 \mu \mathrm{g} / \mathrm{mL}$, apigenin achieved remarkable activity at lower concentration of $3.12 \mu \mathrm{g} / \mathrm{mL}$ (Cushnie et al. 2003; Joung et al. 2016). However, in the study of Su et al. (2014) apigenin along with scutellarein and chlorogenic acid, appeared inactive towards numerous clinical and reference MRSA isolates, as well as against methicillin-sensitive $S$. aureus with MICs higher than $2000 \mu \mathrm{g} / \mathrm{mL}$. Notable anti-MRSA activity has been reported for jaceosidin which bear a $-\mathrm{OCH}_{3}$ group at 5- and 3' positions (Barnes et al. 2013), indicating that other methoxylated flavones from C. calcitrapa extracts may be potential inhibitors of MRSA and might contributed to the EtOAc extract activity, whereas they observed with highest intensities. Furthermore, nepetin a flavonoid with $-\mathrm{OCH}_{3}$ group at $\mathrm{C}-6$ has been reported for inhibition of clinical isolate of MRSA (Talib et al. 2012). Nepetin was also found with highest intensity in EtOAc extract within our study. To the best of our knowledge there are no reports for antimicrobial activity of jaceidin and centaureidin against methicillinresistant S. aureus. Skupińska et al. (2017) reported that the most active inhibitors of E. coli and S. aureus had a -OH groups at 5- and 7-positions and methoxy group at $\mathrm{C}-4^{\prime}$, such as kaempferide with $\mathrm{MIC}_{50}$ values of $22.7 \mu \mathrm{g} /$ $\mathrm{mL}$ and $8.5 \mu \mathrm{g} / \mathrm{mL}$, respectively. Also, authors reported that kaempferol was inactive against $E$. coli, while in the study of Wu et al. (2013) it was reactive with $\mathrm{MIC}_{50}$ value of $25 \mu \mathrm{g} / \mathrm{mL}$, whereas authors indicated that hydroxyl group at C-4' are necessary for the activity. In this study, kaempferide and kaempferol were more abundant in $\mathrm{Me}_{2} \mathrm{CO}$ extract, while eupatorin and centaureidin, which also bear a $-\mathrm{OCH}_{3}$ group at $\mathrm{C}-4^{\prime}$ were prevalent in EtOAc extract and might be responsible for its stronger activity on E. coli and S. aureus than $\mathrm{Me}_{2} \mathrm{CO}$ extract. In the study of Akroum et al. (2009) notable antimicrobial activity of kaempferol 3-O-glucoside and quercetin 3-O-glucoside has been recorded against $S$. aureus and $E$. coli with MIC values from $65-95 \mu \mathrm{g} / \mathrm{mL}$. In line with that, flavonoid glycosides may be one of the compounds that enhance activity of extracts, especially of $\mathrm{Me}_{2} \mathrm{CO}$ extract, whereas they observed in the highest concentrations. Barnes et al. (2013) reported that activity of jaceosidin towards E. coli was higher than $128 \mu \mathrm{g} / \mathrm{mL}$. Also, Allison et al. (2017) reported that antimicrobial activity of jaceosidin and jaceidin against a wild-type strain of $E$. coli was higher than $100 \mu \mathrm{M}$ (which corresponds to values of $33.03 \mu \mathrm{g} /$ $\mathrm{mL}$ and $36.03 \mu \mathrm{g} / \mathrm{mL}$, respectively). Sánchez-Maldonado et al. (2011) have been investigated structure-activity relationship of phenolic acids and found that lipophilicity has a greater impact on antimicrobial activity of hydroxybenzoic acids, than of hydroxycinnamic acids, whose lipophilicity is already established with properties of their side chains. Increasing number of hydroxyl groups decreased activity of hydroxybenzoic acid and when a $-\mathrm{OH}$ groups were substituted with methoxyl groups lipophilicity increased, which also enhanced the activity. $P$-hydroxybenzoic acid with only one $-\mathrm{OH}$ group achieved better inhibitor activity towards $E$. coli (MIC was $0.12 \mu \mathrm{g} / \mathrm{mL}$ ) than more hydroxylated hydroxybenzoic acids. Generally, hydroxycinnamic acid achieved better activity towards $E$. coli with lower MIC values. Due to various methods used and different susceptibility of bacterial strains, results of antimicrobial activity of phenolics in the literature are often dissimilar. However, antimicrobial activity of certain phenolics, which are identified as constituents of $C$. calcitrapa extracts, has been reported towards the same strains used in this study. Gallic, caffeic, $p$-coumaric and chlorogenic acid exhibited significant inhibitor activity against $S$. aureus and MRSA (Rivero-Cruz, 2008; Özçelik et al. 2011; Dimkić et al, 2016). It was shown that flavonoids exhibit better activity than phenolic acids against $E$. coli (Rivero-Cruz, 2008; 
Liu et al. 2013; Wu et al. 2013; Skupińska et al. 2017). In our study, this link is also visible through the presence of flavonoids and phenolic acids in the extracts, whereas methoxylated flavonoids found with highest intensities in EtOAc extract, which expressed the best activity towards E. coli. In the study of Ravn et al. (1989) aesculetin was the most active among tested compounds against $A$. tumefaciens and P. syringae with MIC value lower than $100 \mu \mathrm{g} / \mathrm{mL}$, while ferulic, caffeic and chlorogenic acid exhibited significantly less activity.

A strong antimicrobial effect of cnicin, mellitensin, salonitenolide, $11 \beta$, 13-dihydroxy-salonitenolide, pinoresinol, $\beta$-amyrin and $\beta$-sitosterol obtained from different sources against $S$. aureus, MRSA and E. coli, has been reported (Bruno et al. 2003; Bachelier et al. 2006; Djeddi et al. 2008b; Rivero-Cruz et al. 2009; Bach et al. 2011; Sen et al. 2012; Zhou et al. 2017). These specialized metabolites have also been detected in C. calcitrapa (Karawya et al. 1975; Al-Easa and Rizk 1992; Marco et al. 1992; Bruno et al. 2013). Also, two chalcones have been reported as constituents of $C$. calcitrapa and although they have not been investigated for their activity, it is worth mentioning that chalcones, generally, possess considerable antimicrobial activity, especially anti-MRSA activity (Dawidar et al. 1989; Alcaraz et al. 2000; Xie et al. 2015). It should be borne in mind here that the role of sesquiterpene lactones in increasing the antioxidant and antimicrobial effect of phenolic compounds is known but not fully clarified (Chadwick et al. 2013). Moghannem et al. (2016) isolated, but not completely determined, a compound from the group of sesquiterpene lactones from $\mathrm{MeOH}$ extract of $C$. calcitrapa leaves and examined its effect on bacterial isolates by using the microdilution method. The MIC $(15.6 \mu \mathrm{g} / \mathrm{mL})$ and MBC $(31.25 \mu \mathrm{g} / \mathrm{mL})$ values against $S$. aureus and $E$. coli were lower in regards to our results obtained with $\mathrm{MeOH}$ extract against the mentioned pathogens. To the best of our knowledge our study presents the first report of antimicrobial activity of C. calcitrapa extracts towards phytopathogenic bacteria. Phytopathogenic bacterial strains showed high sensitivity toward tested extracts. It has been reported that phenolic acids with a longer alkyl chain have a higher affinity for lipid bilayers, which may explain high susceptibility of Gram-negative bacteria (Borges et al. 2013). Moreover, hydrophobic character of methoxylated flavonoids may be responsible for the best activity of EtOAc extract against phytopathogenic bacteria, since they were observed with highest intensities in this extract.

In comparison of the total yield of quantified phenolics and prevalence of phenolics through their intensities per extract with accomplished MIC results, it can be seen that EtOAc extract, with the highest representation of methoxylated flavonoids, had the best antimicrobial effect. We can assume that other specialized metabolites such as sesquiterpene lactones, which are known to have strong antimicrobial activity, are also found in a high concentration of EtOAc extract and contributed to overall extract activity. This assumption is based on the work of Van Beek et al. (1990) who identified EtOAc extract as the best one for isolating sesquiterpene lactones. Also, the best anti-MRSA activity of $\mathrm{Me}_{2} \mathrm{CO}$ extract might be due to a greater portion of the polyhydroxylated flavonoids, since they are in the highest concentrations in this extract. Since it is known that chemical structures of flavonoids and phenolic acids, different substitutions, and different mechanisms of action due to their structural variability are responsible for antibacterial activity (Shin et al. 2018), it could be assumed that synergistic effect of the present compounds is responsible for the strong activity of the extracts. This assumption is based on the studies whereas quercetin and luteolin in combination expressed better activity than they were tested alone against different MRSA and $S$. aureus strains (Su et al. 2014; Usman et al. 2016).

As far as we know, this is the first report for cytotoxicity of $C$. calcitrapa extracts on the MRC- 5 cell line. ErolDayi et al. (2011) have reported cytotoxicity for $\mathrm{MeOH}$ extract of C. calcitrapa leaves on Vero and Hela cells, whereas $\mathrm{MeOH}$ extract was less toxic on MRC-5 cells. Similar results showed that cytotoxicity of $\mathrm{MeOH}$ extract of $C$. ainetensis against human normal intestinal cells was negligible with the survival rate over 90\% (El-Najjar et al. 2008). EtOAc extract of several Centaurea species exhibited similar toxicity on MRC-5 cells (Faschingbauer, 2019), which is in accordance with the results in this study. However, cytotoxic effects of extracts from different Centaurea species have been more investigated on cancer cells, than on healthy cell lines. Some flavonoids have been studied for their cytotoxicity on both cell lines. Csapi et al. (2010) indicated that Chl (chloroform) extract of C. arenaria (M. B. ex Willd.) showed the highest cytotoxic activity against several cancer cell lines. Moreover, they considered that flavonoids, sesquiterpene lactones and lignans are responsible for cytotoxicity. Beutler et al. (1998) examined cytotoxicity of flavones with different substitutions and reported that flavones with the best cytotoxic activity were one with a - $\mathrm{OH}$ groups at 5 - and $3^{\prime}$ positions, as well as methoxyl groups at $\mathrm{C}-3$ and $\mathrm{C}-4^{\prime}$. According to some authors, interrelation of substituents on A-ring is also important for the flavonoids cytotoxicity (Lopez-Lazaro et al. 2002). As previously mentioned, all identified flavones in this study were 5- hydroxylated, while some of them fully satisfy these structure-activity correlations such as centaureidin and eupatorin. Centaureidin has been reported as extremely cytotoxic compound towards breast, cervical and skin cancer cell lines 
with submicromolar $\mathrm{IC}_{50}$ values, while eupatorin and apigenin had expressed strong cytotoxicity against the same cell lines, but at higher concentrations (Forgo et al. 2012). Also, eupatorin and scutellarein achieved highly antiproliferative activity on MDA-MB-468 (breast carcinoma), but notably less on MCF-10A normal breast cell line with $\mathrm{IC}_{50}$ values of $50 \mu \mathrm{M}$ and $42 \mu \mathrm{M}$, respectively (Androutsopoulos et al. 2008, 2009). Kaempferide has been reported as considerably cytotoxic on several cancer cell lines and non-toxic on human fibroblast (Nath et al. 2015). Cytotoxicity of apigenin, luteolin and chlorogenic acid towards MRC- 5 cells has been reported, with $\mathrm{IC}_{50}$ values higher than $100 \mu \mathrm{M}, 80 \mu \mathrm{M}$ and $1.97 \pm 0.11 \mathrm{mM}$, respectively (Csupor-Löffler et al. 2011; Burgos-Morón et al. 2012; Masraksa et al. 2020). Nevertheless, jaceosidin was more toxic on normal endometrial cells than on cancer cells (Lee et al. 2013). IC $_{50}$ values of eupatorin and nepetin against Vero cells were higher than $500 \mu \mathrm{g} /$ $\mathrm{mL}$ and $103.54 \pm 2.82 \mu \mathrm{g} / \mathrm{mL}$, respectively (Talib et al. 2012; Beer et al. 2016). Thus, different cell lines are more or less sensitive to the investigated phenols, and while they are toxic on cancer cell lines at low concentrations, on normal cell lines they exhibit this activity at high concentrations, furthermore they are considered non-toxic. Considering the rich phenolic profile of tested extracts, possible explanation for showed cytotoxicity at the highest tested concentrations may lay in the fact that phenolic compounds can act as pro-oxidants. When acting as pro-oxidant agents, phenolic compounds are capable of increasing the cellular level of reactive oxygen species (ROS) to cytotoxic levels, consequently leading to the cell death (Martín-Cordero et al. 2012). Also, higher toxicity of $C$. calcitrapa extracts on MRC-5 cells might be due to synergistic activity of present compounds in the extracts. Moreover, the highest intensities of centaureidin, eupatorin and jaceosidin in EtOAc extract probably contributed to the overall extract toxicity. In conclusion, UHPLC-Orbitrap MS revealed the rich phenolic profile of C. calcitrapa with highest intensities of apigenin, eupatorin, nepetin, jaceosidin, chrysoeriol and chlorogenic acid. To the best of our knowledge, this is the first report of centaureidin, jaceidin, kaempferide, nepetin, flavonoid glycosides, phenolic acids and their esters as constituents of $C$. calcitrapa. The EtOAc extract had the best antimicrobial activity. Certainly, correlation between phenolic compound content and the antimicrobial potential of the extracts was confirmed. The most sensitive human pathogen was methicillin-resistant $S$. aureus, which is probably due to a great deal of polyhydroxylated flavones with anti-MRSA activity. Higher sensitivity of phytopathogenic bacteria onto EtOAc extract, compared to others, is probably influenced by hydrophobic properties of methoxylated flavonoids, among others. All tested extracts, which showed the lowest toxicity but at the same time strong antimicrobial activity, can serve as a potential source of natural new compounds against human pathogenic strains, but also against significant phytopathogenic bacterial isolates. To the best of our knowledge, this is the first report of an evaluation of $C$. calcitrapa extracts against phytopathogenic and certain human pathogenic strains, and determination of their potential toxicity on normal human fetal lung fibroblasts. The future studies might include tests of synergism and cytotoxicity of major standard compounds individually and in combinations, as well as to provide us information of the endophytic microbial population from C. calcitrapa, which could be used in biological control together with the plant extracts in combat against most specific phytopathogens.

\section{Supplementary information}

Supplementary information accompanies this paper at https://doi. org/10.1186/s13568-020-01120-5.

Additional file 1: Table S1. Quantified phenolics and their yield (mg/kg) in C. calcitrapa extracts. Table S2. Proposed metabolites and $\mathrm{m} / \mathrm{z}$ peak areas in five different C. calcitrapa extracts using UPLC-MS/MS4 analysis.

\section{Acknowledgements}

This work was supported by the Ministry of Education, Science and Technological Development of the Republic of Serbia (Grant Nos. 451-03-68/202014/200178, 451-03-68/2020-14/200007 and 451-03-68/2020-14/200168). The funding bodies had no role in the study design, data collection and analysis, the decision to publish or the preparation of the manuscript.

\section{Authors' contributions}

All authors conceived the experimental design and study conception. Material preparation, data collection and analysis were performed by ID, MP, MG, UG, $P R$, and PJ. The first draft of the manuscript was written by ID, MP, MG, and PJ and all authors commented on all later versions of the manuscript. Funding acquisition: ID, SS, and PJ. Supervision: ID, and PJ. All authors read and approved the final manuscript.

\section{Funding}

This work was supported by the Ministry of Education, Science and Technological Development of the Republic of Serbia (Grant Nos. 451-03-68/202014/200178, 451-03-68/2020-14/200007 and 451-03-68/2020-14/200168).

\section{Ethics approval and consent to participate}

This article does not contain any studies with human participants or animals performed by any of the authors.

\section{Consent for publication}

All authors consent for the manuscript to be published.

\section{Competing interests}

The authors declare that they have no competing interests.

\section{Author details}

${ }^{1}$ Faculty of Biology, University of Belgrade, Studentski trg 16, 11000 Belgrade, Serbia. ${ }^{2}$ Department of Plant Physiology, Institute for Biological Research "Siniša Stanković", National Institute of Republic of Serbia, University of Belgrade, Bulevar despota Stefana 142, 11060 Belgrade, Serbia. ${ }^{3}$ Faculty of Chemistry, University of Belgrade, Studentski trg 12-16, 11000 Belgrade, Serbia. 
Received: 21 September 2020 Accepted: 5 October 2020

Published online: 12 October 2020

\section{References}

AAT Bioquest, Inc. (2009) Quest Graph ${ }^{\mathrm{TM}}$ IC50 Calculator. https://www.aatbi o.com/tools/ic50-calculator

Akroum S, Bendjeddou D, Satta D, Lalaoui K (2009) Antibacterial activity and acute toxicity effect of flavonoids extracted from Mentha longifolia. AEJSR 4:93-96

Alcaraz LE, Blanco SE, Puig ON, Tomas F, Ferretti FH (2000) Antibacterial activity of flavonoids against methicillin-resistant Staphylococcus aureus strains. J Theor Biol 205:231-240. https://doi.org/10.1006/jtbi.2000.2062

Al-Easa HS, Rizk AM (1992) Constituents of Centaurea species. Qatar Univ Sci J 12:27-57

Allison BJ, Allenby MC, Bryant SS, Min JE, Hieromnimon M, Joyner PM (2017) Antibacterial activity of fractions from three Chumash medicinal plant extracts and in vitro inhibition of the enzyme enoyl reductase by the flavonoid jaceosidin. Nat Prod Res 31:707-712. https://doi. org/10.1080/14786419.2016.1217201

Androutsopoulos V, Arroo RR, Hall JF, Surichan S, Potter GA (2008) Antiproliferative and cytostatic effects of the natural product eupatorin on MDAMB-468 human breast cancer cells due to CYP1-mediated metabolism. Breast Cancer Res. https://doi.org/10.1186/bcr2090

Androutsopoulos VP, Ruparelia K, Arroo RR, Tsatsakis AM, Spandidos DA (2009) CYP1-mediated antiproliferative activity of dietary flavonoids in MDA-MB-468 breast cancer cells. Toxicology 264:162-170. https://doi. org/10.1016/j.tox.2009.07.023

Arif R, Küpeli E, Ergun F (2004) The biological activity of Centaurea L. species. Gazi Univ J Sci 17:149-164

Ayad R, Ababsa ZE, Belfadel FZ, Akkal S, León F, Brouard I, Medjroubi K (2012) Phytochemical and biological activity of Algerian Centaurea melitensis. Int J Med Arom Plants 2:151-154

Azzouzi D, Bioud K, Demirtas I, Gul F, Sarri D, Benayache S, Benayache F, Mekkiou R (2016) Phenolic profile and antioxidant activity of Centaurea choulettiana Pomel (Asteraceae) extracts. Comb Chem High Throughput Screen 19:841-846. https://doi.org/10.2174/1574888X116661611020 92319

Bach SM, Fortuna MA, Attarian R, De Trimarco JT, Catalán CA, Av-Gay Y, Bach H (2011) Antibacterial and cytotoxic activities of the sesquiterpene lactones cnicin and onopordopicrin. Nat Prod Commun 6:163-166. https://doi. org/10.1177/1934578X1100600202

Bachelier A, Mayer R, Klein CD (2006) Sesquiterpene lactones are potent and irreversible inhibitors of the antibacterial target enzyme MurA. Bioorganic Med Chem Lett 16:5605-5609. https://doi.org/10.1016/j. bmcl.2006.08.021

Bakr RO, Mohamed SAEH, Ayoub N (2016) Phenolic profile of Centaurea aegyptiaca L. growing in Egypt and its cytotoxic and antiviral activities. Afr J Tradit Complement Altern Med 13:135-143. https://doi.org/10.21010/ ajtcam.v13i6.19

Banjanac T, Dragićević M, Šiler B, Gašić U, Bohanec B, Nestorović Živković J, Trifunović S, Mišić D (2017) Chemodiversity of two closely related tetraploid Centaurium species and their hexaploid hybrid: Metabolomic search for high-resolution taxonomic classifiers. Phytochemistry 140:27-44. https://doi.org/10.1016/j.phytochem.2017.04.005

Barnes EC, Kavanagh AM, Ramu S, Blaskovich MA, Cooper MA, Davis RA (2013) Antibacterial serrulatane diterpenes from the Australian native plant Eremophila microtheca. Phytochemistry 93:162-169. https://doi. org/10.1016/j.phytochem.2013.02.021

Beer MF, Frank FM, Elso OG, Bivona AE, Cerny N, Giberti G, Malchiodi EL, Martino VS, Alonso MR, Sülsen VP, Cazorla SI (2016) Trypanocidal and leishmanicidal activities of flavonoids isolated from Stevia satureiifolia var. satureiifolia. Pharm Biol 54:2188-2195. https://doi.org/10.3109/13880 209.2016.1150304

Beutler JA, Hamel E, Vlietinck AJ, Haemers A, Rajan P, Roitman JN, Cardellina JH II, Boyd MR (1998) Structure-activity requirements for flavone cytotoxicity and binding to tubulin. J Med Chem 41:2333-2338. https://doi. org/10.1021/jm970842h
Borges A, Ferreira C, Saavedra MJ, Simões M (2013) Antibacterial activity and mode of action of ferulic and gallic acids against pathogenic bacteria. Microb Drug Resist 19:256-265. https://doi.org/10.1089/mdr.2012.0244

Bruno M, Rosselli S, Maggio A, Raccuglia RA, Napolitano F, Senatore F (2003) Antibacterial evaluation of cnicin and some natural and semisynthetic analogues. Planta Med 69:277-281. https://doi. org/10.1055/s-2003-38491

Bruno M, Bancheva S, Rosselli S, Maggio A (2013) Sesquiterpenoids in subtribe Centaureinae (Cass.) Dumort (tribe Cardueae, Asteraceae): Distribution, 13C NMR spectral data and biological properties. Phytochemistry 95:19-93. https://doi.org/10.1016/j.phytochem.2013.07.002

Burgos-Morón E, Calderón-Montaño JM, Orta ML, Pastor N, Pérez-Guerrero C, Austin C, Mateos S, López-Lázaro M (2012) The coffee constituent chlorogenic acid induces cellular DNA damage and formation of topoisomerase I-and II-DNA complexes in cells. J Agric Food Chem 60:7384-7391. https://doi.org/10.1021/jf300999e

Chadwick M, Trewin H, Gawthrop F, Wagstaff C (2013) Sesquiterpenoids lactones: benefits to plants and people. Int J Mol Sci 14:12780-12805. https://doi.org/10.3390/ijms140612780

Csapi B, Hajdú Z, Zupkó I, Berényi Á, Forgo P, Szabó P, Hohmann J (2010) Bioactivity-guided isolation of antiproliferative compounds from Centaurea arenaria. Phytother Res 24:1664-1669. https://doi.org/10.1002/ ptr.3187

Csupor D, Blazso G, Balogh A, Hohmann J (2010) The traditional Hungarian medicinal plant Centaurea sadleriana Janka accelerates wound healing in rats. J Ethnopharmacol 127:193-195. https://doi.org/10.1016/j. jep.2009.09.049

Csupor-Löffler B, Hajdú Z, Zupkó I, Molnár J, Forgo P, Vasas A, Kele Z, Hohmann J (2011) Antiproliferative constituents of the roots of Conyza canadensis. Planta Med 77:1183-1188. https://doi.org/10.1055/s-0030-1270714

Cushnie TPT, Hamilton VES, Lamb AJ (2003) Assessment of the antibacterial activity of selected flavonoids and consideration of discrepancies between previous reports. Microbiol Res 158:281-289. https://doi. org/10.1078/0944-5013-00206

Dawidar AM, Metwally MA, Abou-Elzahab M, Abdel-Mogib M (1989) Chemical constituents of two Centaurea species. Pharmazie 44:735-736

Dimkić I, Ristivojević P, Janakiev T, Berić T, Trifković J, Milojković-Opsenica D, Stanković S (2016) Phenolic profiles and antimicrobial activity of various plant resins as potential botanical sources of Serbian propolis. Ind Crops Prod 94:856-871. https://doi.org/10.1016/j.indcrop.2016.09.065

Djeddi S, Argyropoulou C, Skaltsa H (2008) Secondary metabolites from Centaurea grisebachii ssp. grisebachii. Biochem Syst Ecol 36:336-339. https:// doi.org/10.1016/j.bse.2007.11.001

Djeddi S, Karioti A, Sokovic M, Koukoulitsa C, Skaltsa H (2008) A novel sesquiterpene lactone from Centaurea pullata: Structure elucidation, antimicrobial activity, and prediction of pharmacokinetic properties. Bioorg Med Chem 16:3725-3731. https://doi.org/10.1016/j.bmc.2008.01.056

Dumlu MU, Gürkan E (2006) A new active compound from Centaurea species. Z Naturforsch C J Biosci 61:44-46. https://doi.org/10.1515/ znc-2006-1-208

El-Najjar N, Dakdouki S, Darwiche N, El-Sabban M, Saliba NA, Gali-Muhtasib H (2008) Anti-colon cancer effects of salograviolide A isolated from Centaurea ainetensis. Oncol Rep 19:897-904. https://doi.org/10.3892/or.19.4.897

Erel SB, Karaalp C, Bedir E, Kaehlig H, GlasI S, Khan S, Krenn L (2011) Secondary metabolites of Centaurea calolepis and evaluation of cnicin for anti-inflammatory, antioxidant, and cytotoxic activities. Pharm Biol 49:840-849. https://doi.org/10.3109/13880209.2010.551538

Erol-Dayi Ö, Pekmez M, Bona M, Aras-Perk A, Arda N (2011) Total phenolic contents, antioxidant activities cytotoxicity of three Centaurea species: C. calcitrapa subsp. calcitrapa, C. ptosimopappa and C. spicata. Free Rad Antiox 1:31-36. https://doi.org/10.5530/ax.2011.2.7

Faschingbauer J (2019) Cytotoxic and cholinesterase inhibitory activity of extracts from selected species of the Centaurea L. genus. Diploma thesis, University of Czech Republic

Flamini G, Antognoli E, Morelli I (2001) Two flavonoids and other compounds from the aerial parts of Centaurea bracteata from Italy. Phytochemistry 57:559-564. https://doi.org/10.1016/50031-9422(01)00066-8

Flamini G, Stoppelli G, Morelli I, Ertugrul K, Dural H, Tugay O, Demirelma H (2004) Secondary metabolites from Centaurea isaurica from Turkey and their chemotaxonomical significance. Biochem Syst Ecol 32:553-557. https://doi.org/10.1016/j.bse.2003.10.014 
Forgo P, Zupkó I, Molnár J, Vasas A, Dombi G, Hohmann J (2012) Bioactivityguided isolation of antiproliferative compounds from Centaurea jacea $\mathrm{L}$. Fitoterapia 83:921-925. https://doi.org/10.1016/j.fitote.2012.04.006

Formisano C, Rigano D, Senatore F, Bancheva S, Maggio A, Rosselli S, Bruno M (2012) Flavonoids in subtribe Centaureinae (Cass.) Dumort. (Tribe Cardueae, Asteraceae): distribution and 13C-NMR spectral data. Chem Biodiverse 9:2096-2158. https://doi.org/10.1002/cbdv.201100208

Gajić M (1975) Centaurea L. In: Josifović M (ed) Flora of SR Serbia, vol 7. Serbian Academy of Science and Arts, Belgrade, pp 229-264

Garcia-Jacas N, Susanna A, Garnatje T, Vilatersana R (2001) Generic delimitation and phylogeny of the subtribe Centaureinae (Asteraceae): a combined nuclear and chloroplast DNA analysis. Ann Bot 87:503-515. https://doi. org/10.1006/anbo.2000.1364

Güven K, Celik S, Uysal I (2005) Antimicrobial activity of Centaurea species. Pharm Biol 43:67-71. https://doi.org/10.1080/13880200590903390

Janaćković P, Rajčević N, Gavrilović M, Novaković J, Giweli A, Stešević D, Marin PD (2019) Essential oil composition of five Artemisia (Compositae) species in regards to chemophenetics. Biochem Syst Ecol 87:103960. https://doi. org/10.1016/j.bse.2019.103960

Javorka S, Csapody V (1975) Iconographia Florae Partis Austro-Orientalis Europae Centralis. Akadémiai Kiadó, Budapest

Joung DK, Lee YS, Han SH, Lee SW, Cha SW, Mun SH, Kong R, Kang OH, Song HJ, Shin DW, Kwon DY (2016) Potentiating activity of luteolin on membrane permeabilizing agent and ATPase inhibitor against methicillinresistant Staphylococcus aureus. Asian Pac J Trop Med 9:19-22. https://doi. org/10.1016/j.apjtm.2015.12.004

Karamenderes C, Konyalioglu S, Khan S, Khan IA (2007) Total phenolic contents, free radical scavenging activities and inhibitory effects on the activation of NF-kappa B of eight Centaurea L. species. Phytother Res 21:488-491. https://doi.org/10.1002/ptr.2097

Karawya MS, Hilal SH, Hifnawy MS, El Hawary SS (1975) Phytochemical study of Centaurea calcitrapa L. growing in Egypt. Egypt J Pharm Sci 16:429-444

Karuppusamy S, Rajasekaran KM (2009) High throughput antibacterial screening of plant extracts by resazurin redox with special reference to medicinal plants of Western Ghats. Glob J Pharmacol 3:63-68

Khammar A, Djeddi S (2012) Pharmacological and biological properties of some Centaurea species. Eur J Sci Res 84:398-416

Kitouni R, Benayache F, Benayache S (2015) Flavonoids of the exudate of Centaurea calcitrapa. Chem Nat Compd 51:762-763. https://doi.org/10.1007/ s10600-015-1404-7

Lee JG, Kim JH, Ahn JH, Lee KT, Baek NI, Choi JH (2013) Jaceosidin, isolated from dietary mugwort (Artemisia princeps), induces G2/M cell cycle arrest by inactivating cdc25C-cdc2 via ATM-Chk1/2 activation. Food Chem Toxicol 55:214-221. https://doi.org/10.1016/j.fct.2012.12.026

Lentini F (2000) The role of ethnobotanics in scientific research. State of ethnobotanical knowledge in Sicily. Fitoterapia 71:S83-S88. https://doi. org/10.1016/S0367-326X(00)00179-9

Litvinenko VI, Bubenchikova VN (1988) Phytochemical study of Centaurea cyanus. Chem Nat Compd 24:672-674. https://doi.org/10.1007/bf005 98180

Liu R, Zhang H, Yuan M, Zhou J, Tu Q, Liu JJ, Wang J (2013) Synthesis and biological evaluation of apigenin derivatives as antibacterial and antiproliferative agents. Molecules 18:11496-11511. https://doi.org/10.3390/ molecules180911496

Lopez-Lazaro M, Galvez M, Martin-Cordero C, Ayuso MJ (2002) Cytotoxicity of flavonoids on cancer cell lines. Structure-activity relationship. Stud Nat Prod Chem 27:891-932. https://doi.org/10.1016/S1572-5995(02)80050-3

Marco JA, Sanz JF, Sancenon F, Susanna A, Rustaiyan A, Saberi M (1992) Sesquiterpene lactones and lignans from Centaurea species. Phytochemistry 31:3527-3530. https://doi.org/10.1016/0031-9422(92)83721-A

Margni M, Rossier D, Crettaz P, Jolliet O (2002) Life cycle impact assessment of pesticides on human health and ecosystems. Agric Ecosyst Environ 93:379-392. https://doi.org/10.1016/S0167-8809(01)00336-X

Martín-Cordero C, León-González AJ, Calderón-Montaño JM, Burgos-Morón E, López-Lázaro M (2012) Pro-oxidant natural products as anticancer agents. Curr Drug Targets 13:1006-1028. https://doi.org/10.2174/1389450128 02009044

Masraksa W, Tanasawet S, Hutamekalin P, Wongtawatchai T, Sukketsiri W (2020) Luteolin attenuates migration and invasion of lung cancer cells via suppressing focal adhesion kinase and non-receptor tyrosine kinase signaling pathway. Nutr Res Pract 14:127-133. https://doi.org/10.4162/ nrp.2020.14.2.127

Moghannem SA, El-Sherbiny GM, Sharaf MH (2016) Antibacterial activity of medicinal plant (Centauraea calcitrapa) against multi-drug resistant bacteria (MDRB). Asia J Appl Microbiol 3:12-25. https://doi.org/10.18488/ journal.33/2016.3.1/33.1.12.25

Mohlenbrock RH (2015) Flowering plants: Asteraceae, Part I. Southern Illinois University Press, Illinois

Mosmann T (1983) Rapid colorimetric assay for cellular growth and survival: application to proliferation and cytotoxicity assays. J Immunol Methods 65:55-63. https://doi.org/10.1016/0022-1759(83)90303-4

Nath LR, Gorantla JN, Joseph SM, Antony J, Thankachan S, Menon DB, Sankar S, Lankalapalli RS, Anto RJ (2015) Kaempferide, the most active among the four flavonoids isolated and characterized from Chromolaena odorata, induces apoptosis in cervical cancer cells while being pharmacologically safe. RSC Adv 5:100912-100922. https://doi.org/10.1039/c5ra19199h

Öksüz S, Ayyildiz H, Johansson C (1984) 6-Methoxylated and C-glycosyl flavonoids from Centaurea species. J Nat Prod 47:902-903. https://doi. org/10.1021/np50035a034

Özçelik B, Kartal M, Orhan I (2011) Cytotoxicity, antiviral and antimicrobial activities of alkaloids, flavonoids, and phenolic acids. Pharm Biol 49:396-402. https://doi.org/10.3109/13880209.2010.519390

Pieroni A, Janiak V, Dürr CM, Lüdeke S, Trachsel E, Heinrich M (2002) In vitro antioxidant activity of non-cultivated vegetables of ethnic Albanians in southern Italy. Phytother Res 16:467-473. https://doi.org/10.1002/ ptr.1243

Ravn H, Andary C, Kovács G, Mølgaard P (1989) Caffeic acid esters as in vitro inhibitors of plant pathogenic bacteria and fungi. Biochem Syst Ecol 17:175-184. https://doi.org/10.1016/0305-1978(89)90076-8

Ristivojević P, Trifković J, Gašić U, Andrić F, Nedić N, Tešić Ž, Milojković-Opsenica D (2015) Ultrahigh-performance Liquid Chromatography and Mass Spectrometry (UHPLC-LTQ/Orbitrap/MS/MS) study of phenolic profile of Serbian poplar type propolis. Phytochem Anal 26:127-136. https://doi. org/10.1002/pca.2544

Rivero-Cruz JF (2008) Antimicrobial compounds isolated from Haematoxylon brasiletto. J Ethnopharmacol 119:99-103. https://doi.org/10.1016/j. jep.2008.06.005

Rivero-Cruz JF, Sánchez-Nieto S, Benítez G, Casimiro X, Ibarra-Alvarado C, Rojas-Molina A, Rivero-Cruz B (2009) Antibacterial compounds isolated from Byrsonima crassifolia. Rev Latinoam de Química 37:155-163

Sánchez-Maldonado AF, Schieber A, Gänzle MG (2011) Structure-function relationships of the antibacterial activity of phenolic acids and their metabolism by lactic acid bacteria. J Appl Microbiol 111:1176-1184. https ://doi.org/10.1111/j.1365-2672.2011.05141.x

Sarker SD, Kumarasamy Y, Shoeb M, Celik S, Yucel E, Middleton M, Nahar L (2005) Antibacterial and antioxidant activities of three Turkish species of the genus Centaurea. Orient Pharm Exp Med 5:246-250. https://doi. org/10.3742/opem.2005.5.3.246

Sen A, Dhavan P, Shukla KK, Singh S, Tejovathi G (2012) Analysis of IR, NMR and antimicrobial activity of $\beta$-sitosterol isolated from Momordica charantia. Sci Secure J Biotechnol 1:9-13

Shin J, Prabhakaran VS, Kim KS (2018) The multi-faceted potential of plantderived metabolites as antimicrobial agents against multidrug-resistant pathogens. Microb Pathog 116:209-214. https://doi.org/10.1016/j.micpa th.2018.01.043

Skupińska M, Stępniak P, Łętowska I, Rychlewski L, Barciszewska M, Barciszewski J, Giel-Pietraszuk M (2017) Natural compounds as inhibitors of tyrosyl-tRNA synthetase. Microb Drug Resist 23:308-320. https://doi. org/10.1089/mdr.2015.0272

Soumia K, Tahar D, Lynda L, Saida B, Chabane C, Hafidha M (2014) Antioxidant and antimicrobial activities of selected medicinal plants from Algeria. J Coast Life Med 2:478-483. https://doi.org/10.12980/JCLM.2.2014A PJTB-2014-0071

Su Y, Ma L, Wen Y, Wang H, Zhang S (2014) Studies of the in vitro antibacterial activities of several polyphenols against clinical isolates of methicillinresistant Staphylococcus aureus. Molecules 19:12630-12639. https://doi. org/10.3390/molecules190812630

Susanna A, Garcia-Jacas N (2007) The tribe Cardueae. In: Kadereit J, Jeffrey C (Eds.) Flowering plants. Eudicots. Asterales. Vol. VIII in Kubitzki K (ed) The families and genera of vascular plants. Springer, Berlin, pp 123-146 
Taamalli A, Arráez-Román D, Abaza L, Iswaldi I, Fernández-Gutiérrez A, Zarrouk M, Segura-Carretero A (2015) LC-MS-based metabolite profiling of methanolic extracts from the medicinal and aromatic species Mentha pulegium and Origanum majorana. Phytochem Anal 26:320-330. https:// doi.org/10.1002/pca.2566

Talib WH, Zarga MHA, Mahasneh AM (2012) Antiproliferative, antimicrobial and apoptosis inducing effects of compounds isolated from Inula viscosa. Molecules 17:3291-3303. https://doi.org/10.3390/molecules17033291

Toribio MS, Oriani SD, Skliar MI (2004) Actividad antimicrobiana de Centaurea solstitialis y Centaurea calcitrapa. Ars Pharm 45:335-341

Usman AM, Khurram M, Khan TA, Faidah HS, Shah ZU, Rahman SU, Haseeb A, Ilyas M, Ullah N, Khayam SMU, Iriti M (2016) Effects of luteolin and quercetin in combination with some conventional antibiotics against methicillin-resistant Staphylococcus aureus. Int J Mol Sci 17:1947. https:// doi.org/10.3390/ijms17111947

Van Beek TA, Maas P, King BM, Leclercq E, Voragen AG, De Groot A (1990) Bitter sesquiterpene lactones from chicory roots. J Agric Food Chem 38:1035-1038. https://doi.org/10.1021/jf00094a026

Wu T, Zang X, He M, Pan S, Xu X (2013) Structure-activity relationship of flavonoids on their anti-Escherichia coli activity and inhibition of DNA gyrase. J Agric Food Chem 61:8185-8190. https://doi.org/10.1021/jf402222v
Xie Y, Yang W, Tang F, Chen X, Ren L (2015) Antibacterial activities of flavonoids: structure-activity relationship and mechanism. Curr Med Chem 22:132-149. https://doi.org/10.2174/0929867321666140916113443

Xu HX, Lee SF (2001) Activity of plant flavonoids against antibiotic-resistant bacteria. Phytother Res 15:39-43. https://doi.org/10.1002/10991573(200102)15:1\%3c39::aid-ptr684\%3e3.0.co;2-r

Zengin G, Zheleva-Dimitrova D, Gevrenova R, Nedialkov P, Mocan A, Ćirić A, Glamočlija J, Soković M, Aktumsek A, Mahomoodally MF (2018) Identification of phenolic components via LC-MS analysis and biological activities of two Centaurea species: C. drabifolia subsp. drabifolia and C. Iycopifolia. J Pharm Biomed Anal 149:436-441. https://doi.org/10.1016/j. jpba.2017.11.045

Zhou H, Ren J, Li Z (2017) Antibacterial activity and mechanism of pinoresinol from Cinnamomum camphora leaves against food-related bacteria. Food Control 79:192-199. https://doi.org/10.1016/j.foodcont.2017.03.041

\section{Publisher's Note}

Springer Nature remains neutral with regard to jurisdictional claims in published maps and institutional affiliations.

\section{Submit your manuscript to a SpringerOpen ${ }^{\circ}$ journal and benefit from:}

- Convenient online submission

- Rigorous peer review

- Open access: articles freely available online

- High visibility within the field

- Retaining the copyright to your article

Submit your next manuscript at $\boldsymbol{\Delta}$ springeropen.com 